\title{
Existence and Stability for the 3D Linearized Constant-Coefficient Incompressible Current-Vortex Sheets
}

\author{
Davide Catania \\ Dipartimento di Matematica, Facoltà di Ingegneria, Università degli Studi di Brescia, Via Valotti 9, 25133 Brescia, Italy \\ Correspondence should be addressed to Davide Catania; davide.catania@ing.unibs.it
}

Received 29 May 2012; Revised 21 December 2012; Accepted 27 December 2012

Academic Editor: Helge Holden

Copyright (C) 2013 Davide Catania. This is an open access article distributed under the Creative Commons Attribution License, which permits unrestricted use, distribution, and reproduction in any medium, provided the original work is properly cited.

\begin{abstract}
We consider the free boundary problem for current-vortex sheets in ideal incompressible magnetohydrodynamics. The problem of current-vortex sheets arises naturally, for instance, in geophysics and astrophysics. We prove the existence of a unique solution to the constant-coefficient linearized problem and an a priori estimate with no loss of derivatives. This is a preliminary result to the study of linearized variable-coefficient current-vortex sheets, a first step to prove the existence of solutions to the nonlinear problem.
\end{abstract}

\section{Introduction}

1.1. The Eulerian Description. Let us consider the equations of magnetohydrodynamics (MHD) governing the motion of a perfectly conducting inviscid incompressible plasma in three-space dimension. In the case of a homogeneous plasma (i.e., the density $\rho$ is a positive constant), the equations in a dimensionless form read

$$
\begin{gathered}
\partial_{t} u+\nabla \cdot(u \otimes u-H \otimes H)+\nabla q=0, \\
\partial_{t} H-\nabla \times(u \times H)=0, \\
\operatorname{div} u=0, \quad \operatorname{div} H=0,
\end{gathered}
$$

where, using $T$ for transposition, $u=\left(u_{1}, u_{2}, u_{3}\right)^{T}$ denotes the plasma velocity, $H=\left(H_{1}, H_{2}, H_{3}\right)^{T}$ is the magnetic field (in Alfvén velocity units), $q=p+|H|^{2} / 2$ is the total pressure, and $p$ is the pressure. For smooth solutions, system (1) can be written in an equivalent form as

$$
\begin{gathered}
\partial_{t} u+(u \cdot \nabla) u-(H \cdot \nabla) H+\nabla q=0, \\
\partial_{t} H+(u \cdot \nabla) H-(H \cdot \nabla) u=0, \\
\operatorname{div} u=0, \quad \operatorname{div} H=0 .
\end{gathered}
$$

We are interested in weak solutions, in a suitable sense, to (1) that are smooth on either side of a smooth hypersurface $\Gamma(t)=\left\{x_{3}=f\left(t, x^{\prime}\right)\right\}$ in $[0, T] \times \Omega$, where $\Omega \subset \mathbb{R}^{3}, x^{\prime}=$ $\left(x_{1}, x_{2}\right)$, and that satisfy suitable jump conditions at each point of the front $\Gamma(t)$. For notational simplicity, we assume that the density is the same constant on either side of $\Gamma(t)$, so that we can take, with no loss of generality, $\rho \equiv 1$. In physical applications, the two densities can be very different, but such a difference intervenes only at the boundary, and it is taken into account by the jump condition for the total pressure (see below), so it does not alter the mathematical techniques applied in this paper.

Let us set $\Omega^{ \pm}(t)=\left\{x_{3} \gtrless f\left(t, x^{\prime}\right)\right\}$, where $\Omega=\Omega^{+}(t) \cup$ $\Omega^{-}(t) \cup \Gamma(t)$; given any function $g$, we set $g^{ \pm}=g$ in $\Omega^{ \pm}(t)$ and denote by $[g]=g_{\mid \Gamma(t)}^{+}-g_{\mid \Gamma(t)}^{-}$the jump across $\Gamma(t)$.

We look for current-vortex sheets solutions, that is, smooth solutions $\left(q^{ \pm}, u^{ \pm}, H^{ \pm}\right)$of $(2)$ in $\Omega^{ \pm}(t)$ such that $\Gamma(t)$ is a tangential discontinuity, namely, the plasma does not flow through the discontinuity front, and the magnetic field is tangent to $\Gamma(t)$, see, for example, Landau and Lifshitz [1]; thus, the boundary conditions take the form

$$
\sigma_{\text {front }}=u^{ \pm} \cdot n, \quad H^{ \pm} \cdot n=0, \quad[q]=0 \quad \text { on } \Gamma(t),
$$

where $n=n(t)$ denotes the outward unit normal on $\partial \Omega^{-}(t)$ and $\sigma_{\text {front }}$ denotes the velocity of propagation of the interface front $\Gamma(t)$. With the given parametrization of $\Gamma(t)$, an equivalent formulation of these jump conditions is

$$
\partial_{t} f=u^{ \pm} \cdot N, \quad H^{ \pm} \cdot N=0, \quad[q]=0 \quad \text { on } \Gamma(t),
$$


with $N:=\left(-\partial_{1} f,-\partial_{2} f, 1\right)^{T}$. Notice that the function $f$ describing the discontinuity front is part of the unknowns of the problem, that is, this is a free boundary problem.

System (2), (4) is supplemented with initial conditions

$$
\begin{gathered}
u^{ \pm}(0, x)=u_{0}^{ \pm}(x), \quad H^{ \pm}(0, x)=H_{0}^{ \pm}(x), \quad x \in \Omega^{ \pm}(0), \\
f\left(0, x^{\prime}\right)=f_{0}\left(x^{\prime}\right), \quad x^{\prime} \in \Gamma(0),
\end{gathered}
$$

where $\operatorname{div} u_{0}^{ \pm}=\operatorname{div} H_{0}^{ \pm}=0$ in $\Omega^{ \pm}(0)$.

Current-vortex sheets have various interesting applications in geophysics and astrophysics. For instance, an accepted model in the literature for the interface region between the unperturbed flows of the interstellar plasma and the supersonic solar wind plasma is given by a current-vortex sheet separating the interstellar plasma compressed at the bow shock from the solar wind plasma compressed at the termination shock, see Ruderman and Fahr [2] and references therein. This current-vortex sheet is called the heliopause and in some sense can be considered as the outer boundary of the solar system. Similarly, the boundary separating the shocked solar wind plasma from the plasma of the magnetosphere of planets equipped with an intrinsic magnetic field (such as the Earth, Jupiter...) is a current-vortex sheet called magnetopause.

In order to prove the local existence of solutions to the current-vortex sheets (nonlinear) problem (2), (4), and (5), it is useful to prove existence for the linearized problem and the strong stability, that is, an a priori estimate for the solutions with no loss of derivatives (see later on). We will perform a further simplification by assuming a linearization around a constant-coefficient basic state. This must be seen as a preliminary step before considering a linearization around a general variable-coefficient basic state, in order to prove existence, uniqueness and stability for the solution to problem (2), (4), and (5) without resorting to a Nash-Moser iteration. Such result would be a rigorous confirmation of the stabilizing effect of the magnetic field on Kelvin-Helmholtz instabilities, which is well known in astrophysics.

In the last years, there has been a renewed interest for the analysis of free interface problems in fluid dynamics, especially for the Euler equations in the vacuum and the water waves problem, see Coutand and Shkoller $[3,4]$ and the references thereinto. This fact has produced different methodologies for obtaining a priori estimates and the proof of the existence of solutions. If the interface moves with the velocity of fluid particles, a natural approach consists in the introduction of the Lagrangian coordinates that reduces the original problem to a new one on a fixed domain. This approach has been recently employed with success in a series of papers by Coutand and Shkoller on the incompressible and compressible Euler equations in the vacuum, see $[3,4]$. However, this method seems hardly applicable to problem (2), (4), and (5).

For incompressible current-vortex sheets, the precise description of the region of weak stability is known, meaning that for states outside this region the problem is ill posed. In this region, Morando et al. [5], for the constant-coefficient linearized equations, have shown an a priori estimate for solutions with loss of regularity with respect to the data. In a subset of the region of weak stability, that is, under a more restrictive stability condition (i.e., (6), introduced in the following), Trakhinin [6] has shown a similar result with no loss of regularity for the solutions to the linearized problem with respect to the source terms, but with loss of derivatives with respect to the coefficients of the problem. Moreover, in a recent paper, Coulombel et al. [7] have shown that the same more restrictive stability condition is sufficient for an a priori estimate of solutions to the nonlinear problem with no loss of regularity (here, space periodicity is assumed). However, in all the previous cases, existence of the solution is missing. Let us note that we are looking for the existence and an a priori estimate with no loss of derivatives for the linearized incompressible current-vortex sheet problem. Reference [6] already provides an estimate with no loss of regularity in the constant-coefficient case, but such a loss manifests for variable coefficients, and existence is not proved. The main novelty of our paper is the existence result; however, we hope that it is possible to extend the techniques here used in order to obtain an a priori estimate with no loss of regularity even for the variable-coefficient linearized problem. We point out that the estimate in [7] is optimal, with no loss of derivatives, but refers to the nonlinear problem and does not help in proving existence for the linearized system.

In [8], Secchi and Trakhinin have established existence and stability for the linearized compressible plasma-vacuum (free boundary) problem. In that case, the interface separates a plasma region governed by the usual MHD equations from a vacuum region with pre-Maxwell dynamics for the magnetic field, as in the case of magnetic confinement in nuclear fusion processes. This work has been employed by Morando et al. [9] to obtain the analogous result for the incompressible problem. However, the techniques used in these papers cannot be easily adapted to current-vortex sheets, since they rely on a secondary symmetrization in the vacuum part that cancels problematic boundary terms and cannot be reproduced in plasma part.

In the present paper, we follow an approach different from the one of Coutand and Shkoller. To reduce our free boundary problem to the fixed domain, we consider a change of variables inspired from Lannes [10] and performed in Secchi and Trakhinin [8]. The control of the function describing the free interface follows from a stability condition introduced by Trakhinin in [11], that is, the strong stability condition

$$
\max \left(\left|H^{+} \times[u]\right|,\left|H^{-} \times[u]\right|\right)<\left|H^{+} \times H^{-}\right|,
$$

which implies in particular that $H^{+} \times H^{-}$is not the zero vector.

We use a compressible approximation and perform some changes of variables in order to obtain a symmetric hyperbolic problem with conditions in a more suitable form, so that we can apply the results by Lax and Phillips [12] and Secchi $[13,14]$ for maximally nonnegative linear symmetric hyperbolic systems with characteristic boundary of constant multiplicity. Another fundamental tool is the so-called secondary symmetrization, which is a linear combination 
of the equations of the hyperbolic system that allows a simplification of the border term necessary to obtain the a priori estimate (see Section 4). By exploiting such techniques, we obtain the existence of a unique solution to the incompressible current-vortex sheets free boundary problem linearized about a suitable constant-coefficient state and show that the solution satisfies a priori estimates with no loss of derivatives with respect to data. As mentioned above, this is a preliminary step in order to prove existence and stability for the current-vortex sheet nonlinear problem.

1.2. The Reference Domain $\Omega$. To avoid using local coordinate charts necessary for arbitrary geometries, and for simplicity, we will assume that the space domain $\Omega$ occupied by the fluid is given by $\Omega=\mathbb{R}^{3}$. We also set

$$
\Omega^{ \pm}:=\Omega \cap\left\{x_{3} \gtrless 0\right\}, \quad \Gamma:=\Omega \cap\left\{x_{3}=0\right\} .
$$

The moving discontinuity front is given by

$$
\Gamma(t):=\Omega \cap\left\{x_{3}=f\left(t, x^{\prime}\right)\right\} .
$$

1.3. An Equivalent Formulation in the Fixed Domain $\Omega$. To reduce the free boundary problem (2), (4), and (5) to the fixed domains $\Omega^{ \pm}$, we introduce a suitable change of variables. In all what follows, $H^{s}(\omega)$ denotes the Sobolev space of order $s$ on a domain $\omega$.

The diffeomorphism that reduces the free boundary problem (2), (4), and (5) to the fixed domains $\Omega^{ \pm}$is given in the following lemma (see [8]).

Lemma 1. Let $m \geq 3$ be an integer. Then, for all $T>0$ and for all $f \in \bigcap_{j=0}^{m-1} \mathscr{C}^{j}\left([0, T] ; H^{m-j-0.5}\left(\mathbb{R}^{2}\right)\right)$ satisfying without loss of generality $\|f\|_{\mathscr{C}\left([0, T] ; H^{2}\left(\mathbb{R}^{2}\right)\right)} \leq 1$, there exists a function

$$
\psi \in \bigcap_{j=0}^{m-1} \mathscr{C}^{j}\left([0, T] ; H^{m-j}\left(\mathbb{R}^{3}\right)\right)
$$

such that the function

$$
\Psi(t, x):=\left(x^{\prime}, x_{3}+\psi(t, x)\right), \quad(t, x) \in[0, T] \times \Omega
$$

defines an $H^{m}$-diffeomorphism of $\Omega$ for all $t \in[0, T]$. Moreover, there holds

$$
\begin{aligned}
& \partial_{t}^{j}(\Psi-I d) \in \mathscr{C}\left([0, T] ; H^{m-j}(\Omega)\right) \quad \text { for } j=0, \ldots, m-1, \\
& \Psi\left(t, x^{\prime}, 0\right)=\left(x^{\prime}, f\left(t, x^{\prime}\right)\right), \quad \partial_{3} \Psi\left(t, x^{\prime}, 0\right)=(0,0,1) .
\end{aligned}
$$

We set

$$
A:=[D \Psi]^{-1} \text { (inverse of the Jacobian matrix), }
$$

$J:=\operatorname{det}[D \Psi]$ (determinant of the Jacobian matrix),

$$
a:=J A \text { (transpose of the cofactor matrix), }
$$

and we compute

$$
\begin{gathered}
A=\left(\begin{array}{ccc}
1 & 0 & 0 \\
0 & 1 & 0 \\
-\frac{\partial_{1} \psi}{J} & -\frac{\partial_{2} \psi}{J} & \frac{1}{J}
\end{array}\right), \quad J=1+\partial_{3} \psi, \\
a=\left(\begin{array}{ccc}
J & 0 & 0 \\
0 & J & 0 \\
-\partial_{1} \psi & -\partial_{2} \psi & 1
\end{array}\right) .
\end{gathered}
$$

Now we may reduce the free boundary problem (2), (4), and (5) to a problem in the fixed domains $\Omega^{ \pm}$by the change of variables (10). Let us set

$$
\begin{gathered}
v^{ \pm}(t, x):=u^{ \pm}(t, \Psi(t, x)), \quad B^{ \pm}(t, x):=H^{ \pm}(t, \Psi(t, x)), \\
Q^{ \pm}(t, x):=q^{ \pm}(t, \Psi(t, x)) .
\end{gathered}
$$

Then, the system (2), (4), and (5) can be reformulated on the fixed reference domains $\Omega^{ \pm}$as

$$
\begin{gathered}
\partial_{t} v^{ \pm}+\left(\widetilde{v}^{ \pm} \cdot \nabla\right) v^{ \pm}-\left(\widetilde{B}^{ \pm} \cdot \nabla\right) B^{ \pm}+A^{T} \nabla Q^{ \pm}=0, \\
\partial_{t} B^{ \pm}+\left(\widetilde{v}^{ \pm} \cdot \nabla\right) B^{ \pm}-\left(\widetilde{B}^{ \pm} \cdot \nabla\right) v^{ \pm}=0, \\
\left(A^{T} \nabla\right) \cdot v^{ \pm}=0, \quad\left(A^{T} \nabla\right) \cdot B^{ \pm}=0, \quad \text { in }[0, T] \times \Omega^{ \pm}, \\
{[Q]=0, \quad \partial_{t} f=v^{ \pm} \cdot N, \quad B^{ \pm} \cdot N=0, \quad \text { on }[0, T] \times \Gamma,} \\
v_{\mid t=0}^{ \pm}=v_{0}^{ \pm}, \quad B_{\mid t=0}^{ \pm}=B_{0}^{ \pm}, \quad \text { on } \Omega^{ \pm}, \\
f_{\mid t=0}=f_{0}, \quad \text { on } \Gamma .
\end{gathered}
$$

In (15), we have set

$$
\begin{gathered}
N:=\left(-\partial_{1} \psi,-\partial_{2} \psi, 1\right)^{T}, \\
\widetilde{v}:=A v-\left(0,0, \frac{\partial_{t} \psi}{J}\right)^{T}=\left(v_{1}, v_{2}, \frac{v \cdot N-\partial_{t} \psi}{J}\right)^{T}, \\
\widetilde{B}:=A B=\left(B_{1}, B_{2}, B \cdot \frac{N}{J}\right)^{T} .
\end{gathered}
$$

Notice that

$$
J=1, \quad N=\left(-\partial_{1} f,-\partial_{2} f, 1\right)^{T}, \quad \widetilde{v}_{3}=\widetilde{B}_{3}=0 \quad \text { on } \Gamma .
$$

We warn the reader that the notation $A^{T}$ is used to denote the transpose of $A$ and has nothing to do with the time interval $[0, T]$ on which the smooth solution is sought; we hope that this does not create any confusion. Vectors are written in columns.

If we set $Q_{T}^{ \pm}=[0, T] \times \Omega^{ \pm}, \omega_{T}=[0, T] \times \Gamma$, and $V^{ \pm}=$ $\left(Q^{ \pm}, v^{ \pm}, B^{ \pm}\right)^{T}$, problem (15) can be written in short form as

$$
\begin{gathered}
\mathbb{P}\left(V^{ \pm}, \psi\right)=\underline{0} \quad \text { in } Q_{T}^{ \pm}, \\
\mathbb{B}\left(V^{ \pm}, f\right)=\underline{0} \quad \text { on } \omega_{T}, \\
\left.\left(v^{ \pm}, B^{ \pm}, f^{ \pm}\right)\right|_{t=0}=\left(v_{0}^{ \pm}, B_{0}^{ \pm}, f_{0}^{ \pm}\right) \quad \text { in }\left(\Omega^{ \pm}\right)^{2} \times \Gamma,
\end{gathered}
$$

where $\underline{0}$ is the zero vector. 


\section{The Linearized Problem}

2.1. Basic State. Let

$$
\left(\widehat{V}^{ \pm}, \widehat{f}\right)
$$

be such that $\widehat{V}^{+}$and $\widehat{V}^{-}$are constant vectors, $\widehat{V}=(\widehat{Q}, \widehat{v}, \widehat{B})^{T}$, and

$$
\widehat{f} \equiv 0 \text {. }
$$

We can then take $\widehat{\psi} \equiv 0$, so that $\widehat{J}=\partial_{3} \widehat{\Psi}_{3}=1+\partial_{3} \widehat{\psi}=$ 1. Similarly, we determine all the "hat" functions as for the corresponding functions obtained for $\left(V^{ \pm}, f\right)$. Let us note, in particular, that $\widehat{A}=\widehat{a}=I_{3}, \widehat{N}^{T}=(0,0,1), \widetilde{\widehat{v}}=\widehat{v}$, and $\widetilde{\widehat{B}}=\widehat{B}$. We assume that

$$
[\widehat{Q}]=0, \quad \widehat{v}_{3}^{ \pm}=\widehat{B}_{3}^{ \pm}=0 .
$$

In particular, $\widehat{Q}^{+}=\widehat{Q}^{-}$. Under these hypotheses, we have that the basic state $\left(\widehat{V}^{ \pm}, \widehat{f}\right)$ is a solution of problem (15).

Moreover, we assume that the basic state satisfies

$$
\max \left(\left|\widehat{B}^{+} \times[\widehat{v}]\right|,\left|\widehat{B}^{-} \times[\widehat{v}]\right|\right)<\left|\widehat{B}^{+} \times \widehat{B}^{-}\right| .
$$

This is the analogue of condition (6) and implies

$$
\left|\widehat{B}^{+} \times \widehat{B}^{-}\right|>0 \text {. }
$$

2.2. Linearized Problem. The linearized equations for (15) are

$$
\begin{aligned}
& \left.\frac{d}{d s} \mathbb{P}\left(V_{s}^{ \pm}, \psi_{s}\right)\right|_{s=0}=\mathrm{F}^{ \pm}, \\
& \left.\frac{d}{d s} \mathbb{B}\left(V_{s}^{ \pm}, f_{s}\right)\right|_{s=0}=g^{ \pm},
\end{aligned}
$$

where $V_{s}=\widehat{V}+s \delta V, f_{s}=\widehat{f}+s \delta f, \psi_{s}=\widehat{\psi}+s \delta \psi$, and $\delta V, \delta f, \delta \psi$ are the unknowns. Here, we consider the source terms in order to make the system inhomogeneous.

If we omit $\delta$ and write explicitly the linearized equations, we get, in $Q_{T}^{ \pm}$,

$$
\begin{gathered}
\partial_{t} v^{ \pm}+\left(\widehat{v}^{ \pm} \cdot \nabla\right) v^{ \pm}-\left(\widehat{B}^{ \pm} \cdot \nabla\right) B^{ \pm}+\nabla Q^{ \pm}=\mathrm{F}_{1}^{ \pm}, \\
\partial_{t} B^{ \pm}+\left(\widehat{v}^{ \pm} \cdot \nabla\right) B^{ \pm}-\left(\widehat{B}^{ \pm} \cdot \nabla\right) v^{ \pm}=\mathrm{F}_{2}^{ \pm}, \\
\operatorname{div} v^{ \pm}=F_{v}^{ \pm}, \\
\operatorname{div} B^{ \pm}=F_{B}^{ \pm} .
\end{gathered}
$$

The linearized boundary conditions are obtained in a similar way (let us recall that we are omitting $\delta$ ):

$$
\begin{gathered}
{[Q]=g_{0},} \\
\partial_{t} f-v_{3}^{ \pm}+\widehat{v}^{\prime \pm} \cdot \nabla^{\prime} f=g_{1}^{ \pm}, \\
-B_{3}^{ \pm}+\widehat{B}^{\prime \pm} \cdot \nabla^{\prime} f=g_{2}^{ \pm}
\end{gathered}
$$

on $\omega_{T}$, where, as usual, if $w=\left(w_{1}, w_{2}, w_{3}\right)^{T}$ is a vector, we set $w^{\prime}=\left(w_{1}, w_{2}\right)^{T}$, and moreover, $\nabla^{\prime}=\left(\partial_{1}, \partial_{2}\right)^{T}$.
2.3. Reduction to Homogeneous Boundary Conditions. Let $\bar{u}^{ \pm}$, $\bar{h}^{ \pm}$denote vector-valued functions which solve, respectively, the elliptic problems

$$
\begin{aligned}
& \operatorname{div} \bar{u}^{ \pm}=F_{v}^{ \pm} \quad \text { in } Q_{T}^{ \pm}, \quad \bar{u}_{3}^{ \pm}=-g_{1}^{ \pm} \quad \text { on } \omega_{T}, \\
& \operatorname{div} \bar{h}^{ \pm}=F_{B}^{ \pm} \quad \text { in } Q_{T}^{ \pm}, \quad \bar{h}_{3}^{ \pm}=-g_{2}^{ \pm} \quad \text { on } \omega_{T}
\end{aligned}
$$

and set $u=v-\bar{u}, h=B-\bar{h}$, and $q=Q-\bar{q}$, where $\bar{q}^{+}=$ $g_{0}$ on $\omega_{T}^{+}$and $\bar{q}^{-}=0$ on $\omega_{T}^{-}$(here, letters $u$ and $q$ represent different variables from the ones introduced in the original formulation (1)).

The equations in (25) can be recast in terms of $(q, u, h)$ :

$$
\begin{gathered}
\partial_{t} u^{ \pm}+\left(\widehat{v}^{ \pm} \cdot \nabla\right) u^{ \pm}-\left(\widehat{B}^{ \pm} \cdot \nabla\right) h^{ \pm}+\nabla q^{ \pm}=F_{1}^{ \pm}, \\
\partial_{t} h^{ \pm}+\left(\widehat{v}^{ \pm} \cdot \nabla\right) h^{ \pm}-\left(\widehat{B}^{ \pm} \cdot \nabla\right) u^{ \pm}=F_{2}^{ \pm},
\end{gathered}
$$$$
\operatorname{div} u^{ \pm}=0, \quad \operatorname{div} h^{ \pm}=0,
$$

with new source terms $F_{i}=\mathrm{F}_{i}+\mathrm{f}_{i}\left(\widehat{v}, \widehat{B}, \nabla \bar{q}, \nabla_{t, x} \bar{u}, \nabla_{t, x} \bar{h}\right), i=$ $0,1,2$, with boundary conditions

$$
\begin{gathered}
{[q]=0} \\
\partial_{t} f-u_{3}^{ \pm}+\widehat{v}^{\prime \pm} \cdot \nabla^{\prime} f=0 \\
-h_{3}^{ \pm}+\widehat{B}^{\prime \pm} \cdot \nabla^{\prime} f=0
\end{gathered}
$$

and initial data

$$
\left.\left(u^{ \pm}, h^{ \pm}, f^{ \pm}\right)\right|_{t=0}=\left(v_{0}^{ \pm}-\bar{u}^{ \pm}(0), B_{0}^{ \pm}-\bar{h}^{ \pm}(0), f_{0}^{ \pm}\right) .
$$

\section{Notations and Results}

3.1. Function Spaces. We denote by $H^{m}=H^{m}(\Omega)$ the usual Sobolev space and by $\|\cdot\|_{m}$ its norm, while $\|\cdot\|$ is simply the norm of $L^{2}=L^{2}(\Omega)$. We introduce a smooth odd function $\sigma=\sigma\left(x_{3}\right)$ defined in $\mathbb{R}$ such that $\sigma\left(x_{3}\right)=x_{3}$ for $0 \leq x_{3} \leq 1 / 2$ and $\sigma\left(x_{3}\right)=1$ for $x_{3} \geq 1$ and set $Z_{1}=\partial_{x_{1}}, Z_{2}=\partial_{x_{2}}$ and $Z_{3}=$ $\sigma \partial_{x_{3}}$. For a multi-index $\alpha=\left(\alpha_{1}, \alpha_{2}, \alpha_{3}\right),|\alpha|=\alpha_{1}+\alpha_{2}+\alpha_{3}$, we set $\partial_{*}^{\alpha}=Z_{1}^{\alpha_{1}} Z_{2}^{\alpha_{2}} Z_{3}^{\alpha_{3}}$,

$$
\begin{gathered}
\|u\|_{m, *}^{2}=\sum_{|\alpha|+2 \beta \leq m}\left\|\partial_{*}^{\alpha} \partial_{x_{3}}^{\beta} u\right\|^{2}, \\
H_{*}^{m}=H_{*}^{m}(\Omega)=\left\{u \in L^{2}:\|u\|_{m, *}<+\infty\right\},
\end{gathered}
$$

where $\beta \geq 0$ is an integer number (see [14-17]).

If $u(t)$, defined on $[0, T]$ with values in $H_{*}^{m}$, is such that $\partial_{t}^{k} u(t) \in H_{*}^{m-k}$ for $k=1,2, \ldots, m$, we set

$$
\begin{gathered}
\|\| u(t)\left\|\left.\right|_{m, *} ^{2}=\sum_{k=0}^{m}\right\| \partial_{t}^{k} u(t) \|_{m-k, *}^{2}, \\
\mathscr{L}_{T}^{p}\left(H_{*}^{m}\right)=\bigcap_{k=0}^{m} W^{k, p}\left(0, T ; H_{*}^{m-k}\right),
\end{gathered}
$$


where $W^{k, p}\left(0, T ; H_{*}^{m-k}\right)$ is the space of functions which are in $L^{p}$ together with the time derivatives up to order $k$ on $[0, T]$, with values in $H_{*}^{m-k}$. The spaces $\mathscr{L}_{T}^{2}\left(H_{*}^{m}\right)$ and $\mathscr{L}_{T}^{\infty}\left(H_{*}^{m}\right)$ are equipped with norms, respectively,

$$
[u]_{m, *, T}^{2}=\int_{0}^{T}\|u(t)\|_{m, *}^{2} d t, \quad\|u\|_{m, *, T}=\sup _{[0, T]}\|u(t)\|_{m, *} .
$$

We define the same function spaces and norms for a general space domain $E$ in the same way; we write, for instance, $\|g\|_{m, * ; E}$ to specify the domain that we are considering.

3.2. Main Result. Now, we are able to state the main theorem. We set $U=(q, u, h)^{T}$ and consider problem

$$
\begin{gathered}
\partial_{t} u^{ \pm}+\left(\widehat{v}^{ \pm} \cdot \nabla\right) u^{ \pm}-\left(\widehat{B}^{ \pm} \cdot \nabla\right) h^{ \pm}+\nabla q^{ \pm}=F_{1}^{ \pm}, \\
\partial_{t} h^{ \pm}+\left(\widehat{v}^{ \pm} \cdot \nabla\right) h^{ \pm}-\left(\widehat{B}^{ \pm} \cdot \nabla\right) u^{ \pm}=F_{2}^{ \pm}, \\
\operatorname{div} u^{ \pm}=0
\end{gathered}
$$

in $Q_{T}^{ \pm}$, with boundary conditions

$$
\begin{gathered}
{[q]=0,} \\
\partial_{t} f-u_{3}^{ \pm}+\widehat{v}^{\prime \pm} \cdot \nabla^{\prime} f=0
\end{gathered}
$$

on $\omega_{T}$, and initial data

$$
\left.\left(u^{ \pm}, h^{ \pm}, f\right)\right|_{t=0}=\left(u_{0}^{ \pm}, h_{0}^{ \pm}, f_{0}\right) \quad \text { in }\left(\Omega^{ \pm}\right)^{2} \times \Gamma,
$$

where

$$
\operatorname{div} u_{0}^{ \pm}=\operatorname{div} h_{0}^{ \pm}=0 \quad \text { on } \Omega^{ \pm} .
$$

Theorem 2. Assume that the constant basic state $\left(\widehat{V}^{ \pm}, \widehat{f}\right)$ satisfies (20), (21), and (22), and moreover, assume that $u_{0}^{ \pm}$, $h_{0}^{ \pm} \in H^{1}\left(\Omega^{ \pm}\right), \operatorname{div} u_{0}^{ \pm}=\operatorname{div} h_{0}^{ \pm}=0$ on $\Omega^{ \pm}$, and $f_{0} \in H^{1 / 2}(\Gamma)$. Assume that $F^{ \pm}=\left(F_{1}^{ \pm}, F_{2}^{ \pm}\right) \in \mathscr{L}_{T}^{2}\left(H_{*}^{1}\left(\Omega^{ \pm}\right)\right)$, $\operatorname{div} F_{2}^{ \pm}=0$ in $Q_{T}^{ \pm}$, and $\left(F_{2}^{ \pm}\right)_{3}=0$ on $[0, T] \times \Gamma$, where $\left(F_{2}^{ \pm}\right)_{3}$ denotes the third component of vector $F_{2}^{ \pm}$. Finally, assume as well the compatibility conditions on $\{t=0\} \times \Gamma$ :

$$
\begin{gathered}
\partial_{t} f_{0}-u_{3}^{ \pm}+\widehat{v}^{\prime \pm} \cdot \nabla^{\prime} f_{0}=0, \\
-h_{3}^{ \pm}+\widehat{B}^{\prime \pm} \cdot \nabla^{\prime} f_{0}=0 .
\end{gathered}
$$

Then there exists a unique solution $\left(U^{ \pm}, f\right)$ to (34), (35), and (36) ( $q^{ \pm}$unique up to additive constants) such that $u^{ \pm}, h^{ \pm} \in L^{\infty}\left([0, T] ; H_{*}^{1}\left(\Omega^{ \pm}\right)\right) \cap W^{1, \infty}\left([0, T] ; L^{2}\left(\Omega^{ \pm}\right)\right), q^{ \pm} \in$ $L^{\infty}\left([0, T] ; \dot{H}^{1}\left(\Omega^{ \pm}\right)\right)\left(\dot{H}^{1}\right.$ is the homogeneous Sobolev space), and $f \in \mathscr{C}^{0}\left([0, T] ; H^{1 / 2}(\Gamma)\right)$; in addition, for each $t \in[0, T]$, the following a priori estimate

$$
\begin{gathered}
\sum_{ \pm}\left(\left\|(u, h)^{ \pm}(t)\right\|_{1, * ; \Omega^{ \pm}}^{2}+\left\|\left(u_{3}, h_{3}\right)^{ \pm}(t)\right\|_{H^{1}\left(\Omega^{ \pm}\right)}^{2}\right. \\
\left.+\left\|\nabla q^{ \pm}(t)\right\|_{L^{2}\left(\Omega^{ \pm}\right)}^{2}\right)+\|f(t)\|_{H^{1 / 2}(\Gamma)}^{2} \\
\leq C e^{C t} \sum_{ \pm}\left(\left\|\left(u_{0}, h_{0}\right)^{ \pm}\right\|_{1, * ; \Omega^{ \pm}}^{2}+\left[F^{ \pm}\right]_{1, *, t ; \Omega^{ \pm}}^{2}\right. \\
\left.+\left\|F_{1}^{ \pm}(0)\right\|_{L^{2}\left(\Omega^{ \pm}\right)}^{2}\right)+\left\|f_{0}\right\|_{H^{1 / 2}(\Gamma)}^{2}
\end{gathered}
$$

is satisfied, where $C=C(\widehat{v}, \widehat{B})$ is a positive constant.

Moreover, one has $\left.\left(u_{3}^{ \pm}, h_{3}^{ \pm}\right)\right|_{\Gamma} \in L^{\infty}\left([0, T] ; H^{1 / 2}(\Gamma)\right)$.

Remark 3. In order to consider the nonlinear problem, it might be useful to have higher regularity estimates, with no loss of derivatives, provided that data are sufficiently regular. The $H^{1}$ regularity can be obtained from the equations for $\operatorname{rot} u$ and $\operatorname{rot} h$, which allow to improve the regularity for the normal derivatives. Taking further derivatives and proceeding as in the proof of Proposition 13, one can expect to get higher regularity. The details are postponed to a future paper.

As for the regularity assumption on $u_{0}^{ \pm}, h_{0}^{ \pm}$, see Section 4.5.

Note that $\operatorname{div} h^{ \pm}=0$ in $Q_{T}^{ \pm}$and $-h_{3}^{ \pm}+\widehat{B}^{\prime \pm} \cdot \nabla^{\prime} f=0$ on $[0, T] \times \Gamma$ follow from (34), (35), and (36) and the assumptions of Theorem 2 and, in particular, from the validity of the restrictions of such equations at time $t=0$. More precisely, we have the following result.

Proposition 4. Under the same assumptions of Theorem 2, the solution $U^{ \pm}$satisfies

$$
\begin{gathered}
\operatorname{div} h^{ \pm}=0 \quad \text { in } Q_{T}^{ \pm}, \\
-h_{3}^{ \pm}+\widehat{B}^{\prime \pm} \cdot \nabla^{\prime} f=0 \quad \text { in } \omega_{T} .
\end{gathered}
$$

Proof. If we take the divergence in the second equation in (34) and use $\operatorname{div} u^{ \pm}=\operatorname{div} F_{2}^{ \pm}=0$ in $Q_{T}^{ \pm}$, we get

$$
\left(\partial_{t}+\widehat{v}^{ \pm} \cdot \nabla\right) \operatorname{div} h^{ \pm}=0,
$$

and hence $\operatorname{div} h^{ \pm}=0$ in $Q_{T}^{ \pm}$, since at time $t=0$, we have $\operatorname{div} h^{ \pm}=\operatorname{div} h_{0}^{ \pm}=0$ on $\Omega^{ \pm}$by assumption.

On the other hand, let us take just the third component of the second equation in

$$
\partial_{t} h_{3}^{ \pm}+\left(\widehat{v}^{\prime \pm} \cdot \nabla^{\prime}\right) h_{3}^{ \pm}-\left(\widehat{B}^{\prime \pm} \cdot \nabla^{\prime}\right) u_{3}^{ \pm}=0,
$$

where we have used $\left(F_{2}^{ \pm}\right)_{3}=0$ and $\widehat{v}_{3}^{ \pm}=\widehat{B}_{3}^{ \pm}=0$. If we substitute $u_{3}^{ \pm}$from the second equation into (35) and observe that $\left(\widehat{B}^{\prime \pm} \cdot \nabla^{\prime}\right)\left(\widehat{v}^{\prime \pm} \cdot \nabla^{\prime} f\right)=\left(\widehat{v}^{ \pm \pm} \cdot \nabla^{\prime}\right)\left(\widehat{B}^{\prime \pm} \cdot \nabla^{\prime} f\right)$, we deduce that

$$
\left(\partial_{t}+\widehat{v}^{\prime \pm} \cdot \nabla^{\prime}\right)\left(h_{3}^{ \pm}-\widehat{B}^{\prime \pm} \cdot \nabla^{\prime} f\right)=0
$$

in $[0, T] \times \Gamma$. Using the third equation in (38), which holds for $t=0$, we conclude that $h_{3}^{ \pm}-\widehat{B}^{\prime \pm} \cdot \nabla^{\prime} f=0$ in $[0, T] \times \Gamma$. 


\section{Proof}

4.1. Hyperbolic Regularization. Now, let us consider the following hyperbolic compressible regularization (see Secchi [18]), where $\varepsilon>0$ :

$$
\begin{aligned}
& \partial_{t} q^{ \pm}+\left(\widehat{v}^{ \pm} \cdot \nabla\right) q^{ \pm}-\varepsilon \widehat{B}^{ \pm} \cdot\left(\partial_{t} h^{ \pm}+\left(\widehat{v}^{ \pm} \cdot \nabla\right) h^{ \pm}\right)+\frac{1}{\varepsilon} \operatorname{div} u^{ \pm}=0 \\
& \partial_{t} u^{ \pm}+\left(\widehat{v}^{ \pm} \cdot \nabla\right) u^{ \pm}-\left(\widehat{B}^{ \pm} \cdot \nabla\right) h^{ \pm}+\frac{1}{\varepsilon} \nabla q^{ \pm}=F_{1}^{ \pm}, \\
& \partial_{t} h^{ \pm}+\left(\widehat{v}^{ \pm} \cdot \nabla\right) h^{ \pm}-\left(\widehat{B}^{ \pm} \cdot \nabla\right) u^{ \pm} \\
& \quad-\varepsilon \widehat{B}^{ \pm}\left\{\partial_{t} q^{ \pm}+(\widehat{v} \cdot \nabla) q^{ \pm}-\varepsilon \widehat{B}^{ \pm} \cdot\left(\partial_{t} h^{ \pm}+\left(\widehat{v}^{ \pm} \cdot \nabla\right) h^{ \pm}\right)\right\} \\
& =F_{2}^{ \pm}
\end{aligned}
$$

with boundary conditions (35).

Here, the solution $U=(q, u, h)^{T}$ depends on $\varepsilon$ but, in this moment, we will not use the more precise notation $U^{\varepsilon}=$ $\left(q^{\varepsilon}, u^{\varepsilon}, h^{\varepsilon}\right)^{T}$ to denote the solution to the previous system (with suitable initial data). Let us note that, at least formally, if we project the system (45), (46), and (47) on the subspace of divergence-free functions, it converges, as $\varepsilon \rightarrow 0$, to system (34) projected on the same subspace; it is sufficient to multiply (45) by $\varepsilon$ and take the limit. We will make this statement more precise in Section 4.7.

Equations (45)-(47) in $Q_{T}^{ \pm}$form a symmetric system:

$$
\begin{aligned}
& \left(\begin{array}{ccc}
1 & \underline{0}^{T} & -\varepsilon\left(\widehat{B}^{ \pm}\right)^{T} \\
\underline{0} & I_{3} & O_{3} \\
-\varepsilon \widehat{B}^{ \pm} & O_{3} & I_{3}+\varepsilon^{2} \widehat{B}^{ \pm} \otimes \widehat{B}^{ \pm}
\end{array}\right) \partial_{t}\left(\begin{array}{c}
q^{ \pm} \\
u^{ \pm} \\
h^{ \pm}
\end{array}\right)+\frac{1}{\varepsilon}\left(\begin{array}{c}
\operatorname{div} u^{ \pm} \\
\nabla q^{ \pm} \\
\underline{0}
\end{array}\right) \\
& +\left(\begin{array}{ccc}
\widehat{v}^{ \pm} \cdot \nabla & \underline{0}^{T} & -\varepsilon\left(\widehat{B}^{ \pm}\right)^{T} \widehat{v}^{ \pm} \cdot \nabla \\
\underline{0} & \widehat{v}^{ \pm} \cdot \nabla & -\widehat{B}^{ \pm} \cdot \nabla \\
-\varepsilon \widehat{B}^{ \pm} \widehat{v}^{ \pm} \cdot \nabla & -\widehat{B}^{ \pm} \cdot \nabla & \left(I_{3}+\varepsilon^{2} \widehat{B}^{ \pm} \otimes \widehat{B}^{ \pm}\right) \widehat{v}^{ \pm} \cdot \nabla
\end{array}\right)\left(\begin{array}{l}
q^{ \pm} \\
u^{ \pm} \\
h^{ \pm}
\end{array}\right) \\
& =\left(\begin{array}{c}
0 \\
F_{1}^{ \pm} \\
F_{2}^{ \pm}
\end{array}\right),
\end{aligned}
$$

or in a compact form,

$$
L^{ \pm} U^{ \pm} \doteq A_{0}^{ \pm} \partial_{t} U^{ \pm}+\sum_{j=1}^{3}\left(A_{j}^{ \pm}+\frac{1}{\varepsilon} \mathscr{E}_{1, j+1}\right) \partial_{j} U^{ \pm}=F^{ \pm}
$$

where each matrix $A_{j}^{ \pm}$is bounded in $\varepsilon$ and $\mathscr{E}_{h, i}=\left(e_{j, k}^{(h, i)}\right)_{1 \leq j, k \leq 7}$, where $e_{j, k}^{(h, i)}=1$ if $(j, k)=(h, i)$ or $(j, k)=(i, h), e_{j, k}^{(h, i)}=0$ otherwise. Notice that $A_{3}^{ \pm}=\mathrm{O}_{7}$ because of (21).
One can easily check that, as $\varepsilon \rightarrow 0$, this system converges to (34), at least formally. Moreover, the system is hyperbolic, since (omitting \pm )

$$
A_{0} U \cdot U=(q-\varepsilon \widehat{B} \cdot h)^{2}+|u|^{2}+|h|^{2}>0
$$

for each $(q, u, h) \neq(0, \underline{0}, \underline{0})$.

4.2. Secondary Symmetrization. This new formulation is obtained by a linear combination of (45), (46), and (47) in the previous step and aims to simplify boundary terms in the following computations (see Remark 5).

More precisely, let us consider the system

$$
\begin{gathered}
(45)+\varepsilon \lambda \widehat{B} \cdot(46)-\frac{\lambda}{\varepsilon} \operatorname{div} h=0, \\
\varepsilon \lambda \widehat{B}(45)+(46)-\lambda(54)=\underline{0}, \\
-\lambda\left(I_{3}+\varepsilon^{2} \widehat{B} \otimes \widehat{B}\right)(46)+(47)=\underline{0}
\end{gathered}
$$

where $\lambda=\lambda^{ \pm}$will be chosen conveniently later on and (54) denotes the equation obtained by substituting (45) into (47), that is,

$$
\partial_{t} h^{ \pm}+\left(\widehat{v}^{ \pm} \cdot \nabla\right) h^{ \pm}-\left(\widehat{B}^{ \pm} \cdot \nabla\right) u^{ \pm}+\widehat{B}^{ \pm} \operatorname{div} u^{ \pm}=F_{2}^{ \pm} .
$$

We get

$$
\begin{aligned}
& \left(d_{t} q-\varepsilon \widehat{B} \cdot d_{t} h+\frac{1}{\varepsilon} \operatorname{div} u\right)+\varepsilon \lambda \widehat{B} \cdot\left\{d_{t} u-(\widehat{B} \cdot \nabla) h+\frac{1}{\varepsilon} \nabla q\right\} \\
& -\frac{\lambda}{\varepsilon} \operatorname{div} h=\varepsilon \lambda \widehat{B} \cdot F_{1}, \\
& \varepsilon \lambda \widehat{B}\left(d_{t} q-\varepsilon \widehat{B} \cdot d_{t} h\right)+d_{t} u-(\widehat{B} \cdot \nabla) h+\frac{1}{\varepsilon} \nabla q \\
& -\lambda\left\{d_{t} h-(\widehat{B} \cdot \nabla) u\right\}=F_{1}-\lambda F_{2}, \\
& -\lambda\left(I_{3}+\varepsilon^{2} \widehat{B} \otimes \widehat{B}\right)\left\{d_{t} u-(\widehat{B} \cdot \nabla) h+\frac{1}{\varepsilon} \nabla q\right\}+d_{t} h-(\widehat{B} \cdot \nabla) u \\
& -\varepsilon \widehat{B}\left(d_{t} q-\varepsilon \widehat{B} \cdot d_{t} h\right)=-\lambda\left(I_{3}+\varepsilon^{2} \widehat{B} \otimes \widehat{B}\right) F_{1}+F_{2},
\end{aligned}
$$

where $d_{t} \doteq \partial_{t}+(\widehat{v} \cdot \nabla)$. This system is symmetric; indeed, the coefficient of $d_{t}$ is

$$
\mathscr{A}_{0}=\left(\begin{array}{ccc}
1 & \varepsilon \lambda \widehat{B}^{T} & -\varepsilon \widehat{B}^{T} \\
\varepsilon \lambda \widehat{B} & I_{3} & -\lambda\left(I_{3}+\varepsilon^{2} \widehat{B} \otimes \widehat{B}\right) \\
-\varepsilon \widehat{B}-\lambda\left(I_{3}+\varepsilon^{2} \widehat{B} \otimes \widehat{B}\right) & I_{3}+\varepsilon^{2} \widehat{B} \otimes \widehat{B}
\end{array}\right),
$$

the singular term is given by

$$
\frac{1}{\varepsilon}\left(\begin{array}{ccc}
0 & \nabla^{T} & -\lambda \nabla^{T} \\
\nabla & O_{3} & O_{3} \\
-\lambda \nabla & O_{3} & O_{3}
\end{array}\right)
$$


and the remaining derivatives are included in

$$
\left(\begin{array}{ccc}
\lambda \widehat{B} \cdot \nabla & \underline{0}^{T} & -\varepsilon \lambda \widehat{B}^{T}(\widehat{B} \cdot \nabla) \\
\underline{0} & \lambda \widehat{B} \cdot \nabla & -\widehat{B} \cdot \nabla \\
-\varepsilon \lambda(\widehat{B} \otimes \widehat{B}) \nabla & -\widehat{B} \cdot \nabla & \lambda\left(I_{3}+\varepsilon^{2} \widehat{B} \otimes \widehat{B}\right)(\widehat{B} \cdot \nabla)
\end{array}\right),
$$

where $\mathrm{I}_{3}$ and $\mathrm{O}_{3}$ denote, respectively, the identity and null $3 \times$ 3 matrix. All these matrices are symmetric, and the system can be recast as

$\mathscr{L}^{ \pm} U^{ \pm} \doteq \mathscr{A}_{0}^{ \pm} \partial_{t} U^{ \pm}+\sum_{j=1}^{3}\left(\mathscr{A}_{j}^{ \pm}+\frac{1}{\varepsilon} \mathscr{E}_{1, j+1}-\frac{\lambda}{\mathcal{E}} \mathscr{E}_{1, j+4}\right) \partial_{j} U^{ \pm}=\mathscr{F}^{ \pm}$

where each matrix $\mathscr{A}_{j}^{ \pm}$is bounded in $\varepsilon$ provided $\lambda^{ \pm}$is bounded and $\mathscr{E}_{h, i}=\left(e_{j, k}^{(h, i)}\right)_{1 \leq j, k \leq 7}$, where $e_{j, k}^{(h, i)}=1$ if $(j, k)=$ $(h, i)$ or $(j, k)=(i, h), e_{j, k}^{(h, i)}=0$ otherwise. Notice that $\mathscr{A}_{3}^{ \pm}=$ $\mathrm{O}_{7}$.

Remark 5. The secondary symmetrization is inspired by [11, 19], which we refer to for further details. The main idea is to consider the following variant of (49), which takes into account the divergence constraint for the magnetic field

$$
D^{ \pm} A_{0}^{ \pm} \partial_{t} U^{ \pm}+\sum_{j=1}^{3} D^{ \pm}\left(A_{j}^{ \pm}+\frac{1}{\varepsilon} \mathscr{E}_{1, j+1}\right) \partial_{j} U^{ \pm}+\lambda^{ \pm} E^{ \pm} \operatorname{div} h^{ \pm}=F^{ \pm}
$$

where the matrix $D^{ \pm}$and the vector $E^{ \pm}$depend on the basic state. $D$ is then chosen in order to obtain $\mathscr{A}_{0}$, whose form is useful to get the energy a priori estimate, while $E$ is chosen to maintain the symmetry of the system. As pointed out in [19] (Remark 5), such a choice is related to the conservation of cross-helicity: $d / d t \int(v \cdot B) d x=0$. On the other hand, one main feature of the secondary symmetrization is that $\lambda^{ \pm}$can be chosen widely arbitrarily, so that in the following we will be able to find a suitable $\lambda^{ \pm}$which simplifies singular boundary terms in the a priori estimate.

The following lemma guarantees that, under our assumptions and a suitable choice of $\lambda^{ \pm}$, the matrices $\mathscr{A}_{0}^{ \pm}$are positive definite and (59) is symmetric hyperbolic.

Lemma 6. If

$$
\left(\lambda^{ \pm}\right)^{2}<\frac{1}{1+\left|\varepsilon \widehat{B}^{ \pm}\right|^{2}}
$$

then the symmetric matrices $\mathscr{A}_{0}^{ \pm}$are positive definite.
Proof. Omitting “ \pm ”, we have

$$
\begin{aligned}
\mathscr{A}_{0} U & \cdot U \\
= & (q+\varepsilon \lambda \widehat{B} \cdot u-\varepsilon \widehat{B} \cdot h) q+\left\{\varepsilon \lambda \widehat{B} q+u-\lambda\left(I_{3}+\varepsilon^{2} \widehat{B} \otimes \widehat{B}\right) h\right\} \cdot u \\
& +\left\{-\varepsilon \widehat{B} q-\lambda\left(I_{3}+\varepsilon^{2} \widehat{B} \otimes \widehat{B}\right) u+\left(I_{3}+\varepsilon^{2} \widehat{B} \otimes \widehat{B}\right) h\right\} \cdot h \\
= & (q-\varepsilon \widehat{B} \cdot h)^{2}+u \cdot u+h \cdot h+2 \varepsilon \lambda \widehat{B} \cdot u(q-\varepsilon \widehat{B} \cdot h)-2 \lambda h \cdot u \\
\geq & (q-\varepsilon \widehat{B} \cdot h+\varepsilon \lambda \widehat{B} \cdot u)^{2}+|h-\lambda u|^{2}+\left(1-\lambda^{2}-\lambda^{2}|\varepsilon \widehat{B}|^{2}\right)|u|^{2} .
\end{aligned}
$$

If

$$
1-\lambda^{2}-\lambda^{2}|\varepsilon \widehat{B}|^{2}>0
$$

that is to say, if

$$
\lambda^{2}<\frac{1}{1+|\varepsilon \widehat{B}|^{2}}
$$

then the matrix $\mathscr{A}_{0}$ is positive definite.

Lemma 7. There exists $\varepsilon_{0}>0$ such that, for each $\left.\left.\varepsilon \in\right] 0, \varepsilon_{0}\right]$, the choice

$$
\lambda^{ \pm}=\widehat{\lambda}^{ \pm} \doteq \frac{[\widehat{v}] \times \widehat{B}^{\mp}}{\left|\widehat{B}^{+} \times \widehat{B}^{-}\right|} \operatorname{sign}\left(\widehat{B}_{1}^{+} \widehat{B}_{2}^{-}-\widehat{B}_{2}^{+} \widehat{B}_{1}^{-}\right)
$$

satisfies (61) and hence makes the matrices $\mathscr{A}_{0}^{ \pm}$positive definite.

Proof. Since

$$
\frac{\left|[\widehat{v}] \times \widehat{B}^{\mp}\right|}{\left|\widehat{B}^{+} \times \widehat{B}^{-}\right|}<1
$$

which follows from (22), we deduce that there exists $\varepsilon_{0}>0$ such that, for each $\varepsilon \in\left[0, \varepsilon_{0}[\right.$, the inequality

$$
\frac{\left|[\widehat{v}] \times \widehat{B}^{\mp}\right|}{\left|\widehat{B}^{+} \times \widehat{B}^{-}\right|} \leq \frac{1}{\sqrt{1+\left|\varepsilon \widehat{B}^{ \pm}\right|^{2}}}
$$

follows, so we conclude that (61) holds true for $\varepsilon$ small enough.

Let us remark that $\hat{\lambda}^{ \pm}$is a constant term independent of $\varepsilon$; moreover, since $\widehat{B}_{3}^{ \pm}=0$, this choice of $\lambda^{ \pm}$is the same as

$$
\widehat{\lambda}^{ \pm}=\frac{\left[\widehat{v}_{1}\right] \widehat{B}_{2}^{\mp}-\left[\widehat{v}_{2}\right] \widehat{B}_{1}^{\mp}}{\widehat{B}_{1}^{+} \widehat{B}_{2}^{-}-\widehat{B}_{2}^{+} \widehat{B}_{1}^{-}}
$$

which will be useful in the following steps. Moreover, this choice is equivalent to

$$
\left[\widehat{v}^{\prime}-\hat{\lambda} \widehat{B}^{\prime}\right]=\underline{0}
$$

In addition, we set

$$
\Delta=\widehat{B}_{1}^{+} \widehat{B}_{2}^{-}-\widehat{B}_{2}^{+} \widehat{B}_{1}^{-}
$$

which is always different from zero thanks to (23). 
4.3. Boundary Conditions in Algebraic Form. The boundary conditions can be recast so that the space derivatives of the front function $f$ do not appear explicitly.

Lemma 8. The boundary conditions (35) imply, on $\omega_{T}$, the boundary conditions

$$
\begin{gathered}
{[q]=0,} \\
{\left[u_{3}-\hat{\lambda} h_{3}\right]=0}
\end{gathered}
$$

and the front equation

$$
\partial_{t} f=u_{3}^{ \pm}+S_{+}^{ \pm} h_{3}^{-}-S_{-}^{ \pm} h_{3}^{+},
$$

with

$$
\begin{gathered}
S_{+}^{ \pm}=\frac{1}{\Delta}\left(\widehat{v}_{1}^{ \pm} \widehat{B}_{2}^{+}-\widehat{v}_{2}^{ \pm} \widehat{B}_{1}^{+}\right), \quad S_{-}^{ \pm}=\frac{1}{\Delta}\left(\widehat{v}_{1}^{ \pm} \widehat{B}_{2}^{-}-\widehat{v}_{2}^{ \pm} \widehat{B}_{1}^{-}\right), \\
\Delta=\widehat{B}_{1}^{+} \widehat{B}_{2}^{-}-\widehat{B}_{2}^{+} \widehat{B}_{1}^{-}
\end{gathered}
$$

Proof. Condition (41) can be recast as

$$
\begin{aligned}
& \widehat{B}_{1}^{+} \partial_{1} f+\widehat{B}_{2}^{+} \partial_{2} f=h_{3}^{+}, \\
& \widehat{B}_{1}^{-} \partial_{1} f+\widehat{B}_{2}^{-} \partial_{2} f=h_{3}^{-} .
\end{aligned}
$$

Since

$$
\Delta=\operatorname{det}\left(\begin{array}{cc}
\widehat{B}_{1}^{+} & \widehat{B}_{2}^{+} \\
\widehat{B}_{1}^{-} & \widehat{B}_{2}^{-}
\end{array}\right) \neq 0
$$

by hypothesis, we can obtain

$$
\begin{gathered}
\partial_{1} f=\frac{1}{\Delta}\left\{h_{3}^{+} \widehat{B}_{2}^{-}-h_{3}^{-} \widehat{B}_{2}^{+}\right\}, \\
\partial_{2} f=-\frac{1}{\Delta}\left\{h_{3}^{+} \widehat{B}_{1}^{-}-h_{3}^{-} \widehat{B}_{1}^{+}\right\} .
\end{gathered}
$$

By substituting in the second equation of (35), we get

$$
\partial_{t} f=u_{3}^{ \pm}+\frac{\widehat{v}_{1}^{ \pm} \widehat{B}_{2}^{+}-\widehat{v}_{2}^{ \pm} \widehat{B}_{1}^{+}}{\Delta} h_{3}^{-}-\frac{\widehat{v}_{1}^{ \pm} \widehat{B}_{2}^{-}-\widehat{v}_{2}^{ \pm} \widehat{B}_{1}^{-}}{\Delta} h_{3}^{+} .
$$
tions

Using the definition of $S_{+}^{ \pm}$and $S_{-}^{ \pm}$, we deduce the condi-

$$
\begin{gathered}
\partial_{t} f=u_{3}^{ \pm}+S_{+}^{ \pm} h_{3}^{-}-S_{-}^{ \pm} h_{3}^{+}, \\
u_{3}^{+}+S_{+}^{+} h_{3}^{-}-S_{-}^{+} h_{3}^{+}=u_{3}^{-}+S_{+}^{-} h_{3}^{-}-S_{-}^{-} h_{3}^{+} .
\end{gathered}
$$

The last condition can be recast as

$$
\left[u_{3}\right]=-\left[S_{+}\right] h_{3}^{-}+\left[S_{-}\right] h_{3}^{+} \text {, }
$$

where

$$
\left[S_{+}\right]=S_{+}^{+}-S_{+}^{-}, \quad\left[S_{-}\right]=S_{-}^{+}-S_{-}^{-} .
$$

Recalling (68), we have

$$
\widehat{\lambda}^{ \pm}=\left[S_{\mp}\right] \text {. }
$$

The previous condition becomes

$$
\left[u_{3}-\widehat{\lambda} h_{3}\right]=0
$$

and the lemma is proved.
4.4. Equivalence of Systems. Obviously, if $\left(U^{\varepsilon}, f^{\varepsilon}\right)$ is a solution to system (49) with boundary conditions (35), then it also satisfies system (59) with conditions (71) (we are assuming the same initial conditions); the converse holds true as well.

Proposition 9. If $U^{\varepsilon}$ is a solution to system (59) with boundary conditions (71), then one can find a unique $f^{\varepsilon}$, so that $\left(U^{\varepsilon}, f^{\varepsilon}\right)$ is a solution to system (49) with boundary conditions (35) and the same initial conditions.

Proof. First, we prove that (49) is satisfied. Since (59) holds, then we have (51), (52), and (53). If we substitute $(54)=(47)$ $+\varepsilon \widehat{B}(45)$ into (52), we get $(46)=\widehat{\lambda}(47)$, and substituting this relation in (53), we deduce

$$
\left(I_{3}-\widehat{\lambda}^{2} I_{3}-\widehat{\lambda}^{2} \varepsilon^{2} \widehat{B} \otimes \widehat{B}\right)(47)=0 .
$$

Since

$$
\operatorname{det}\left(I_{3}-\widehat{\lambda}^{2} I_{3}-\widehat{\lambda}^{2} \varepsilon^{2} \widehat{B} \otimes \widehat{B}\right)=\left(1-\hat{\lambda}^{2}\right)^{2}\left(1-\hat{\lambda}^{2}-\widehat{\lambda}^{2} \varepsilon^{2}|\widehat{B}|^{2}\right) \neq 0
$$

thanks to $(61)$, then we get $(47)=\underline{0}$ and consequently $(46)=$ $\widehat{\lambda}(47)=\underline{0}$.

From (51), in order to prove (45), it is now sufficient to establish that $\operatorname{div} h^{\varepsilon}=0$, since we already know that $(46)=\underline{0}$. With this aim, we substitute (51) into (47) and get

$$
\partial_{t} h^{\varepsilon}+(\widehat{v} \cdot \nabla) h^{\varepsilon}-\widehat{\lambda} \widehat{B} \operatorname{div} h^{\varepsilon}+\widehat{B} \operatorname{div} u^{\varepsilon}-(\widehat{B} \cdot \nabla) u^{\varepsilon}=F_{2} \text {. }
$$

Taking the divergence of the above equation, exploiting the assumption $\operatorname{div} F_{2}=0$ and the fact that $\widehat{v}, \widehat{B}, \widehat{\lambda}$ are piecewise constant, and simplifying, we deduce that

$$
\left(\partial_{t}+(\widehat{v}-\hat{\lambda} \widehat{B}) \cdot \nabla\right) \operatorname{div} h^{\varepsilon}=0 .
$$

Since $\operatorname{div} h^{\varepsilon}=0$ in $\{t=0\} \times \Omega^{ \pm}$by assumption on the initial data, we conclude that $\operatorname{div} h^{\varepsilon}=0$ in $Q_{T}^{ \pm}$, so that $(45)=0$ from (51). Then (45), (46), and (47) are satisfied, which means that (49) holds.

Now, we have to prove (35). Condition $\left[q^{\varepsilon}\right]=0$ on $\omega_{T}$ obviously holds, since we have the same condition in (71). We need to prove that there exists $f^{\varepsilon}$ so that $\partial_{t} f^{\varepsilon}+\widehat{v}^{\prime \pm} \cdot \nabla^{\prime} f^{\varepsilon}=u_{3}^{\varepsilon, \pm}$ on $\omega_{T}$. Given $u_{3}^{\varepsilon, \pm}$ and $h_{3}^{\varepsilon, \pm}$, let us define $f^{ \pm}$as the solutions of

$$
\begin{gathered}
\partial_{t} f^{ \pm}+\widehat{v}^{\prime \pm} \cdot \nabla^{\prime} f^{ \pm}=u_{3}^{\varepsilon, \pm} \text { in } \omega_{T}, \\
\left.f^{ \pm}\right|_{t=0}=f_{0} \quad \text { on } \Gamma .
\end{gathered}
$$

Thus, for the completion of the proof of (35), it is sufficient to show that $f^{+}=f^{-}$in $\omega_{T}$. Proceeding as in the proof of Proposition 4 , we first deduce $h_{3}^{\varepsilon, \pm}=\left(\widehat{B}^{\prime \pm} \cdot \nabla^{\prime}\right) f^{ \pm}$in $\omega_{T}$. Let us compute

$$
u_{3}^{\varepsilon, \pm}-\widehat{\lambda}^{ \pm} h_{3}^{\varepsilon, \pm}=\partial_{t} f^{ \pm}+\left(\widehat{v}^{\prime \pm} \cdot \nabla^{\prime}\right) f^{ \pm}-\widehat{\lambda}^{ \pm}\left(\widehat{B}^{\prime \pm} \cdot \nabla^{\prime}\right) f^{ \pm} \text {. }
$$


Since $\left[u_{3}^{\varepsilon}-\hat{\lambda} h_{3}^{\varepsilon}\right]=0$ by $(71)$, we obtain

$$
\left(\partial_{t}+\left(\widehat{v}^{\prime+}-\hat{\lambda}^{+} \widehat{B}^{\prime+}\right) \cdot \nabla^{\prime}\right) f^{+}=\left(\partial_{t}+\left(\widehat{v}^{\prime-}-\widehat{\lambda}^{-} \widehat{B}^{\prime-}\right) \cdot \nabla^{\prime}\right) f^{-} .
$$

Thanks to (69), we have $\widehat{w}^{\prime} \doteq \widehat{v}^{\prime+}-\widehat{\lambda}^{+} \widehat{B}^{\prime+}=\widehat{v}^{\prime-}-\widehat{\lambda}^{-} \widehat{B}^{\prime-}$, and hence,

$$
\begin{gathered}
\left(\partial_{t}+\widehat{w}^{\prime} \cdot \nabla^{\prime}\right) f^{+}=\left(\partial_{t}+\widehat{w}^{\prime} \cdot \nabla^{\prime}\right) f^{-} \text {in } \omega_{T}, \\
\left.f^{ \pm}\right|_{t=0}=f_{0} \quad \text { on } \Gamma .
\end{gathered}
$$

Thus, we can take $f^{\mathcal{E}} \doteq f^{+}=f^{-}$in $\omega_{T}$, and (35) holds.

4.5. Existence of the Approximate Solution. Now, consider the system (59) with the boundary conditions (71) and the initial condition $U^{\mathcal{\varepsilon} \pm}(0)=U_{0}^{ \pm} \doteq\left(0, u_{0}^{ \pm}, h_{0}^{ \pm}\right)^{T}$. This system can be written as

$$
\begin{gathered}
\mathscr{L} U^{\varepsilon}=\mathscr{F} \quad \text { in }[0, T] \times \Omega, \\
\mathscr{M} U^{\varepsilon}=\underline{0} \quad \text { in }[0, T] \times \Gamma, \\
U^{\varepsilon}(0)=U_{0} \quad \text { in } \Omega,
\end{gathered}
$$

where we have set $U^{\varepsilon}=\left(U^{\varepsilon,+}, U^{\varepsilon,-}\right)^{T}$ and similarly for the other quantities; moreover, we set $\left\|U^{\varepsilon}\right\|_{\Omega}=\left\|U^{\varepsilon,+}\right\|_{\Omega^{+}}+\left\|U^{\mathcal{\varepsilon},-}\right\|_{\Omega^{-}}$ and so on. Notice that $\mathscr{M} U^{\mathcal{E}}=\underline{0}$ corresponds to the boundary conditions (71).

Let us note that, since $\left.q_{0}^{ \pm} \doteq q^{\varepsilon, \pm}\right|_{t=0} \equiv 0$, the condition on $\Gamma$ given by $\left[q_{0}\right]=0$ is automatically satisfied. Moreover, if we denote by $\left.\left(\partial_{t} q^{ \pm}, \partial_{t} u^{ \pm}, \partial_{t} h^{ \pm}\right)\right|_{t=0}$ the homonymous quantities in (91) evaluated at time $t=0$ and calculated in terms of the initial data, from the equations and the hypotheses on $q_{0}^{ \pm}, u_{0}^{ \pm}$, $h_{0}^{ \pm}$, we get the condition $\left.\left(\partial_{t} q^{ \pm}, \partial_{t} u^{ \pm}, \partial_{t} h^{ \pm}\right)\right|_{t=0} \in L^{2}\left(\Omega^{ \pm}\right)$. Let us note that the hypothesis $u_{0}^{ \pm}, h_{0}^{ \pm}$in $H^{1}\left(\Omega^{ \pm}\right)$, rather than simply in $H_{*}^{1}\left(\Omega^{ \pm}\right)$, is needed exactly here. We have the following result.

Proposition 10. There exists $\varepsilon_{0}>0$ so that, for each $\left.\varepsilon \in\right] 0$, $\left.\varepsilon_{0}\right]$, there exists a unique solution $U^{\varepsilon} \in \mathscr{C}^{0}\left([0, T] ; H_{*}^{1}(\Omega)\right) \cap$ $\mathscr{C}^{1}\left([0, T] ; L^{2}(\Omega)\right)$ to problem (91); moreover, one has

$$
\|\| U^{\mathcal{\varepsilon}}(t)\|\|_{1, * ; \Omega} \leq C_{\varepsilon} e^{C_{\varepsilon} t}\left(\left\|U_{0}^{\varepsilon}\right\|_{1, * ; \Omega}+[\mathscr{F}]_{1, *, t ; \Omega}\right)
$$

for each $t \in[0, T]$ and a suitable $C_{\varepsilon}>0$.

Proof. The boundary matrix of system (91) is given by

$$
\mathscr{A}_{v}=\left(\begin{array}{ll}
\mathscr{A}_{v}^{+} & O_{7} \\
O_{7} & \mathscr{A}_{v}^{-}
\end{array}\right),
$$

where $\mathscr{A}_{v}^{ \pm}=\mp\left(\mathscr{A}_{3}^{ \pm}+(1 / \varepsilon) \mathscr{E}_{1,4}-\left(\hat{\lambda}^{ \pm} / \varepsilon\right) \mathscr{E}_{1,7}\right)$. The matrix $\mathscr{A}_{v}$ is singular with constant rank (indeed, $\mathscr{A}_{3}^{ \pm}$is the null matrix) and, moreover, ker $\mathscr{M}$ is maximally nonnegative for $\mathscr{A}_{v}$, that is to say,

$$
\mathscr{A}_{v} U^{\varepsilon} \cdot U^{\varepsilon} \geq 0
$$

for each $U^{\varepsilon} \in \operatorname{ker} \mathscr{M}$ (nonnegativity) and such a property does not hold in any other vector space properly containing ker $\mathscr{M}$ (maximality). Indeed, we have

$$
\begin{aligned}
\left.\mathscr{A}_{\nu}\right|_{\Gamma} U^{\varepsilon} \cdot U^{\varepsilon} & =-\left.\mathscr{A}_{\nu}^{+}\right|_{\Gamma} U^{\varepsilon,+} \cdot U^{\varepsilon,+}+\left.\mathscr{A}_{\nu}^{-}\right|_{\Gamma} U^{\varepsilon,-} \cdot U^{\varepsilon,-} \\
& =-\frac{2}{\varepsilon}\left\{\left[q^{\varepsilon}\right]\left(u_{3}^{\varepsilon,+}-\hat{\lambda}^{+} h_{3}^{\varepsilon,+}\right)+q^{\varepsilon,-}\left[u_{3}^{\varepsilon}-\widehat{\lambda} h_{3}^{\varepsilon}\right]\right\} \\
& =0
\end{aligned}
$$

thanks to $\left[q^{\varepsilon}\right]=0$ and $\left[u_{3}^{\varepsilon}-\hat{\lambda} h_{3}^{\varepsilon}\right]=0$ on ker $\mathscr{M}$. Moreover, let us observe that both $\mathscr{A}_{v}^{+}$and $\mathscr{A}_{v}^{-}$have exactly one negative eigenvalue, so we have the same number of conditions defining $\operatorname{ker} \mathscr{M}$ and negative eigenvalues, that is, 2 ; this implies maximality.

Thus, we can apply the results in Lax and Phillips [12] (existence in $L^{2}$, see as well Friedrichs [20] for the noncharacteristic case) and Secchi $[13,14]$ (regularity in $H_{*}^{m}$ ), and deduce the existence of a unique global solution $U^{\varepsilon} \epsilon$ $\mathscr{C}^{0}\left([0, T] ; H_{*}^{1}(\Omega)\right) \cap \mathscr{C}^{1}\left([0, T] ; L^{2}(\Omega)\right)$ to problem (91) such that (92) holds for each $t \in[0, T]$, provided $\varepsilon>0$ is sufficiently small.

Remark 11. Let us note that, in the previous result, the smallness of $\varepsilon$ is necessary in order to have $\mathscr{A}_{0}^{ \pm}$positive definite.

We need to have a number of boundary conditions in (71), that is, defining ker $\mathscr{M}$, equal to the number of negative eigenvalues of $\mathscr{A}_{v}$, that is to say, 2 , in order to have maximality.

Corollary 12. There exists $\varepsilon_{0}>0$, so that, for each $\left.\left.\varepsilon \in\right] 0, \varepsilon_{0}\right]$, there exists a unique solution $\left(U^{\varepsilon}, f^{\varepsilon}\right)$ to problem (49), (35), and (36) with $\left.q^{\varepsilon, \pm}\right|_{t=0} \equiv 0$ such that $U^{\mathcal{\varepsilon}} \in \mathscr{C}^{0}\left([0, T] ; H_{*}^{1}(\Omega)\right) \cap$ $\mathscr{C}^{1}\left([0, T] ; L^{2}(\Omega)\right),\left.\left(q, u_{3}, h_{3}\right)^{\varepsilon, \pm}\right|_{\omega_{T}} \in \mathscr{C}^{0}\left([0, T] ; H^{1 / 2}(\Gamma)\right)$ and $f^{\mathcal{E}} \in \mathscr{C}^{0}\left([0, T] ; H^{1 / 2}(\Gamma)\right)$; moreover, one has

$$
\begin{aligned}
& \left\|U^{\varepsilon}(t)\right\|\left\|_{1, * ; \Omega}+\right\| f^{\varepsilon}(t) \|_{H^{1 / 2}(\Gamma)} \\
& \quad \leq C_{\varepsilon} e^{C_{\varepsilon} t}\left(\left\|U_{0}^{\varepsilon}\right\|_{1, * ; \Omega}+[\mathscr{F}]_{1, *, t ; \Omega}\right)+\left\|f_{0}\right\|_{H^{1 / 2}(\Gamma)}
\end{aligned}
$$

for each $t \in[0, T]$ and a suitable $C_{\varepsilon}>0$.

Proof. Problem (91) with $\left.f^{\varepsilon}\right|_{t=0}=f_{0}$ on $\Gamma$ is a different way to say that (59), (71), and (36) with $\left.q^{\varepsilon, \pm}\right|_{t=0} \equiv 0$ are satisfied. Using Propositions 9 and 10 , we get the result for $U^{\varepsilon}$. Let us note that, since $u^{\varepsilon, \pm} \in \mathscr{C}^{0}\left([0, T] ; H_{*}^{1}(\Omega)\right)$ and the normal component $u_{3}^{\varepsilon, \pm}$ at the boundary is noncharacteristic, then the trace $\left.u_{3}^{\varepsilon, \pm}\right|_{\omega_{T}}$ is in $\mathscr{C}^{0}\left([0, T] ; H^{1 / 2}(\Gamma)\right)$, as shown in Secchi $[13,14]$ and Shizuta [21]. In particular, $\partial_{3} u_{3}^{\varepsilon}$ can be estimated using the other terms appearing in (45), so that we have

$$
\left\|u_{3}^{\varepsilon}(t)\right\|_{H^{1}(\Omega)} \leq C \mid\left\|U^{\varepsilon}(t)\right\|_{1, * ; \Omega} .
$$

The same holds true for $q^{\varepsilon}$ as well, for the same reason $\left(\partial_{3} q^{\varepsilon}\right.$ is obtained from (46)), and for $\partial_{3} h_{3}^{\varepsilon}$, since $\operatorname{div} h^{\varepsilon}=0$ is bounded (this follows from (54) proceeding similarly as in Proposition 4; note that, taking the divergence, the terms 
containing $u^{\varepsilon}$ cancel together). Thus, from the transport equation (87), we find $f^{\mathcal{E}} \in \mathscr{C}^{0}\left([0, T] ; H^{1 / 2}(\Gamma)\right)$ and

$$
\begin{aligned}
& \left\|f^{\varepsilon}(t)\right\|_{H^{1 / 2}(\Gamma)} \\
& \leq\left\|f_{0}\right\|_{H^{1 / 2}(\Gamma)}+t\left\|u_{3}^{\varepsilon,+}\right\|_{L^{\infty}\left(0, t ; H^{1 / 2}(\Gamma)\right)} \\
& \leq\left\|f_{0}\right\|_{H^{1 / 2}(\Gamma)}+C t\left\|u_{3}^{\varepsilon,+}\right\|_{L^{\infty}\left(0, t ; H^{1}\left(\Omega^{+}\right)\right)} \\
& \leq\left\|f_{0}\right\|_{H^{1 / 2}(\Gamma)}+C_{\varepsilon} e^{C_{\varepsilon} t} \sum_{ \pm}\left(\left\|U_{0}^{ \pm}\right\|_{1, * ; \Omega^{ \pm}}+\left[F^{ \pm}\right]_{1, *, t ; \Omega^{ \pm}}\right) .
\end{aligned}
$$

This concludes the proof.

4.6. A Priori Energy Estimate Uniform in $\varepsilon$. Since estimate (96) depends on $\varepsilon$, we cannot use it when passing to the limit as $\varepsilon \rightarrow 0$. We can deduce the following a priori estimate for $\left(U^{\varepsilon, \pm}, f^{\varepsilon}\right)$, which is an $L^{2}$-estimate uniform in $\varepsilon$, useful to pass to the limit as $\varepsilon \rightarrow 0$. We recall that the choice $\lambda=\hat{\lambda}$ makes $\mathscr{A}_{0}^{ \pm}$positive definite, and hence, if we fix $\varepsilon_{0}>0$ sufficiently small, there exists a suitable real number $\left.\left.\alpha_{0} \in\right] 0,1\right]$, so that $\mathscr{A}_{0}^{ \pm} \geq \alpha_{0} I_{7}$ for each $\left.\left.\varepsilon \in\right] 0, \varepsilon_{0}\right]$.

Proposition 13. There exists $\varepsilon_{0}>0$, such that, for each $\left.\varepsilon \in\right] 0$, $\left.\varepsilon_{0}\right]$, the a priori estimate

$$
\begin{gathered}
\sum_{ \pm}\left(\left\|U^{\varepsilon, \pm}(t)\right\|_{1, * ; \Omega^{ \pm}}^{2}+\left\|\left(u_{3}, h_{3}\right)^{\varepsilon, \pm}(t)\right\|_{H^{1}\left(\Omega^{ \pm}\right)}^{2}\right)+\left\|f^{\varepsilon}(t)\right\|_{H^{1 / 2}(\Gamma)}^{2} \\
\leq \frac{C}{\alpha_{0}} e^{C t / \alpha_{0}} \sum_{ \pm}\left(\left\|U_{0}^{ \pm}\right\|_{1, * ; \Omega^{ \pm}}^{2}+\left[F^{ \pm}\right]_{1, *, t ; \Omega^{ \pm}}^{2}\right)+\left\|f_{0}\right\|_{H^{1 / 2}(\Gamma)}^{2}
\end{gathered}
$$

holds for each $t \in[0, T]$ and for a suitable $C>0$ independent of $\varepsilon$, where $U^{\varepsilon}$ is the solution to problem (49), (35), and (36) and $f^{\varepsilon}$ satisfies (87) with initial condition $\left.f^{\varepsilon}\right|_{t=0}=f_{0}$.

Proof. Let us take the scalar product of (59), which is equivalent to (49), with $U^{\mathcal{\varepsilon}, \pm}$ and integrate over $Q_{t}^{ \pm}$(recall that $\lambda^{ \pm}=$ $\hat{\lambda}^{ \pm}$); integrating by parts the terms with spatial derivatives of $U^{\mathcal{E}, \pm}$, exploiting the fact that the coefficient matrices are constant, and recalling that the boundary matrix is given by $\mathscr{A}_{v}^{ \pm}=\mp\left(\mathscr{A}_{3}^{ \pm}+(1 / \varepsilon) \mathscr{E}_{1,4}-\left(\hat{\lambda}^{ \pm} / \varepsilon\right) \mathscr{E}_{1,7}\right)=\mp\left((1 / \varepsilon) \mathscr{E}_{1,4}-\left(\widehat{\lambda}^{ \pm} /\right.\right.$ $\left.\varepsilon) \mathscr{E}_{1,7}\right)$, since $\mathscr{A}_{3}^{ \pm}=O_{7}$, we get

$$
\begin{aligned}
& \int_{Q_{t}^{ \pm}} \mathscr{A}_{0}^{ \pm} \partial_{t} U^{\varepsilon, \pm} \cdot U^{\varepsilon, \pm} d x d t \\
& \mp \frac{1}{2 \varepsilon} \int_{\omega_{t}}\left(\mathscr{E}_{1,4}-\hat{\lambda}^{ \pm} \mathscr{E}_{1,7}\right) U^{\varepsilon, \pm} \cdot U^{\varepsilon, \pm} d x^{\prime} d t \\
& =\frac{1}{2} \int_{Q_{t}^{ \pm}}\left(\sum_{j=1}^{3} \partial_{j}\left(\mathscr{A}_{j}^{ \pm}+\frac{1}{\varepsilon} \mathscr{E}_{1, j+1}-\frac{\hat{\lambda}^{ \pm}}{\varepsilon} \mathscr{E}_{1, j+4}\right)\right) U^{\varepsilon, \pm} \cdot U^{\varepsilon, \pm} d x d t \\
& +\int_{Q_{t}^{ \pm}} \mathscr{F}^{ \pm} \cdot U^{\varepsilon, \pm} d x d t \\
& =\int_{Q_{t}^{ \pm}} \mathscr{F}^{ \pm} \cdot U^{\varepsilon, \pm} d x d t
\end{aligned}
$$

where sum over \pm is assumed. Let us note that, since the basic state is piecewise constant, then $\mathscr{A}_{j}^{ \pm}, \mathscr{E}_{h, k}$ and $\widehat{\lambda}^{ \pm}$are piecewise constant as well; thus,

$$
\sum_{j=1}^{3} \partial_{j}\left(\mathscr{A}_{j}^{ \pm}+\frac{1}{\varepsilon} \mathscr{E}_{1, j+1}-\frac{\hat{\lambda}^{ \pm}}{\varepsilon} \mathscr{E}_{1, j+4}\right)=0
$$

in $Q_{t}^{ \pm}$. Moreover,

$$
\begin{aligned}
\sum_{ \pm} & \mp \frac{1}{2 \varepsilon} \int_{\omega_{t}}\left(\mathscr{E}_{1,4}-\widehat{\lambda}^{ \pm} \mathscr{E}_{1,7}\right) U^{\varepsilon, \pm} \cdot U^{\varepsilon, \pm} d x^{\prime} d t \\
& =-\frac{1}{\varepsilon} \int_{\omega_{t}}\left\{\left[q^{\varepsilon}\right]\left(u_{3}^{\varepsilon,+}-\widehat{\lambda}^{+} h_{3}^{\varepsilon,+}\right)+q^{\varepsilon,-}\left[u_{3}^{\varepsilon}-\hat{\lambda} h_{3}^{\varepsilon}\right]\right\} d x^{\prime} d t \\
& =0,
\end{aligned}
$$

thanks to the boundary conditions $\left[q^{\varepsilon}\right]=0$ and $\left[u_{3}^{\varepsilon}-\widehat{\lambda} h_{3}^{\varepsilon}\right]=$ 0 , that hold in $\omega_{t}$.

We deduce the equality

$$
\sum_{ \pm} \int_{Q_{t}^{ \pm}} \mathscr{A}_{0}^{ \pm} \partial_{t} U^{\varepsilon, \pm} \cdot U^{\varepsilon, \pm} d x d \tau=\sum_{ \pm} \int_{Q_{t}^{ \pm}} \mathscr{F}^{ \pm} \cdot U^{\varepsilon, \pm} d x d \tau .
$$

For the left-hand side, integrating by parts in time and exploiting the symmetry of $\mathscr{A}_{0}^{ \pm}$, we have

$$
\begin{aligned}
\int_{\Omega^{ \pm}} \int_{0}^{t} \mathscr{A}_{0}^{ \pm} \partial_{t} U^{\mathcal{\varepsilon}, \pm} \cdot U^{\mathcal{\varepsilon} \pm} d \tau d x= & \frac{1}{2} \int_{\Omega^{ \pm}} \mathscr{A}_{0}^{ \pm} U^{\mathcal{\varepsilon}, \pm}(t) \cdot U^{\mathcal{\varepsilon}, \pm}(t) d x \\
& -\frac{1}{2} \int_{\Omega^{ \pm}} \mathscr{A}_{0}^{ \pm} U_{0}^{ \pm} \cdot U_{0}^{ \pm} d x .
\end{aligned}
$$

By applying the Cauchy-Schwarz inequality in the right-hand side, we obtain

$$
\begin{aligned}
& \sum_{ \pm} \int_{\Omega^{ \pm}} \mathscr{A}_{0}^{ \pm} U^{\varepsilon, \pm}(t) \cdot U^{\varepsilon, \pm}(t) d x \\
& \quad \leq \int_{0}^{t} \sum_{ \pm}\left\|U^{\varepsilon, \pm}(\tau)\right\|_{L^{2}\left(\Omega^{ \pm}\right)}^{2} d \tau+\sum_{ \pm} \int_{\Omega^{ \pm}} \mathscr{A}_{0}^{ \pm} U_{0}^{ \pm} \cdot U_{0}^{ \pm} d x \\
& \quad+\sum_{ \pm} \int_{0}^{t}\left\|\mathscr{F}^{ \pm}(\tau)\right\|_{L^{2}\left(\Omega^{ \pm}\right)}^{2} d \tau
\end{aligned}
$$

and hence, by exploiting $\mathscr{A}_{0}^{ \pm} \geq \alpha_{0} I_{7}$,

$$
\begin{aligned}
\sum_{ \pm}\left\|U^{\varepsilon, \pm}(t)\right\|_{L^{2}\left(\Omega^{ \pm}\right)}^{2} & \\
\leq & \frac{1}{\alpha_{0}} \int_{0}^{t} \sum_{ \pm}\left\|U^{\varepsilon, \pm}(\tau)\right\|_{L^{2}\left(\Omega^{ \pm}\right)}^{2} d \tau \\
& +\frac{1}{\alpha_{0}} \sum_{ \pm}\left(C\left\|U_{0}^{ \pm}\right\|_{L^{2}\left(\Omega^{ \pm}\right)}^{2}+\int_{0}^{t}\left\|\mathscr{F}^{ \pm}(\tau)\right\|_{L^{2}\left(\Omega^{ \pm}\right)}^{2} d \tau\right) .
\end{aligned}
$$


Through a standard application of Grönwall's lemma, we deduce that

$$
\begin{aligned}
& \sum_{ \pm}\left\|U^{\varepsilon, \pm}(t)\right\|_{L^{2}\left(\Omega^{ \pm}\right)}^{2} \\
& \quad \leq \frac{C e^{t / \alpha_{0}}}{\alpha_{0}} \sum_{ \pm}\left(\left\|U_{0}^{ \pm}\right\|_{L^{2}\left(\Omega^{ \pm}\right)}^{2}+\int_{0}^{t}\left\|F^{ \pm}(\tau)\right\|_{L^{2}\left(\Omega^{ \pm}\right)}^{2} d \tau\right) .
\end{aligned}
$$

In the same way, we can apply a tangential derivative $Z=$ $\partial_{t}, \partial_{x_{1}}, \partial_{x_{2}}$ to (59) and (35), take the scalar product with $Z U^{\mathcal{E}, \pm}$, and proceed as before to obtain

$$
\begin{aligned}
& \sum_{ \pm}\left\|Z U^{\varepsilon, \pm}(t)\right\|_{L^{2}\left(\Omega^{ \pm}\right)}^{2} \\
& \quad \leq \frac{C e^{t / \alpha_{0}}}{\alpha_{0}} \sum_{ \pm}\left(\left\|Z U_{0}^{ \pm}\right\|_{L^{2}\left(\Omega^{ \pm}\right)}^{2}+\int_{0}^{t}\left\|Z F^{ \pm}(\tau)\right\|_{L^{2}\left(\Omega^{ \pm}\right)}^{2} d \tau\right) .
\end{aligned}
$$

It remains to find an estimate for $Z_{3} U^{\mathcal{\varepsilon}, \pm}$, where $Z_{3}=$ $\sigma \partial_{3}=\sigma \partial_{x_{3}}$. If we apply $\sigma \partial_{3}$ to (59) and recall that $\mathscr{A}_{3}^{ \pm}=O_{7}$, we get

$$
\begin{gathered}
\mathscr{A}_{0}^{ \pm} \partial_{t}\left(\sigma \partial_{3} U^{\varepsilon, \pm}\right)+\sum_{j=1}^{3}\left(\mathscr{A}_{j}^{ \pm}+\frac{1}{\varepsilon} \mathscr{E}_{1, j+1}-\frac{\hat{\lambda}^{ \pm}}{\varepsilon} \mathscr{E}_{1, j+4}\right) \partial_{j}\left(\sigma \partial_{3} U^{\varepsilon, \pm}\right) \\
=\sigma \partial_{3} \mathscr{F}^{ \pm}+\left(\frac{1}{\varepsilon} \mathscr{E}_{1,4}-\frac{\hat{\lambda}^{ \pm}}{\varepsilon} \mathscr{E}_{1,7}\right) \sigma^{\prime} \partial_{3} U^{\varepsilon, \pm} .
\end{gathered}
$$

Testing by $\sigma \partial_{3} U^{\varepsilon, \pm}$, summing up terms with plus and minus sign, and simplifying as before, we deduce that

$$
\begin{aligned}
& \sum_{ \pm} \int_{Q_{t}^{ \pm}} \mathscr{A}_{0}^{ \pm} \partial_{t}\left(\sigma \partial_{3} U^{\varepsilon, \pm}\right) \cdot \sigma \partial_{3} U^{\varepsilon, \pm} d x d t \\
& \quad=\sum_{ \pm} \int_{Q_{t}^{ \pm}} \sigma \partial_{3} \mathscr{F}^{ \pm} \cdot \sigma \partial_{3} U^{\varepsilon, \pm} d x d t+\sum_{ \pm} I^{ \pm}
\end{aligned}
$$

where

$$
I^{ \pm}=\frac{1}{\varepsilon} \int_{Q_{t}^{ \pm}}\left(\mathscr{E}_{1,4}-\hat{\lambda}^{ \pm} \mathscr{E}_{1,7}\right) \sigma^{\prime} \partial_{3} U^{\varepsilon, \pm} \cdot \sigma \partial_{3} U^{\varepsilon, \pm} d x d t,
$$

so that we get

$$
\begin{aligned}
& \frac{\alpha_{0}}{2} \sum_{ \pm}\left\|Z_{3} U^{\varepsilon, \pm}(t)\right\|_{L^{2}\left(\Omega^{ \pm}\right)}^{2} \\
& \quad \leq \sum_{ \pm}\left(C\left\|Z_{3} U_{0}^{ \pm}\right\|_{L^{2}\left(\Omega^{ \pm}\right)}^{2}+\left|I^{ \pm}\right|\right) \\
& \quad+\sum_{ \pm} \frac{1}{2} \int_{0}^{t}\left(\left\|Z_{3} U^{\varepsilon, \pm}(\tau)\right\|_{L^{2}\left(\Omega^{ \pm}\right)}^{2}+\left\|Z_{3} \mathscr{F}^{ \pm}(\tau)\right\|_{L^{2}\left(\Omega^{ \pm}\right)}^{2}\right) d \tau
\end{aligned}
$$

by applying the Cauchy-Schwarz inequality. Therefore, we need to estimate $\left|I^{ \pm}\right|$:

$$
\begin{aligned}
\left|I^{ \pm}\right| & =\left|\int_{Q_{t}^{ \pm}} 2 \sigma^{\prime} \frac{\partial_{3} q^{\varepsilon, \pm}}{\varepsilon}\left(\sigma \partial_{3} u^{\varepsilon, \pm}-\hat{\lambda}^{ \pm} \sigma \partial_{3} h^{\varepsilon, \pm}\right) d x d \tau\right| \\
& \leq C \int_{Q_{t}^{ \pm}} \frac{\partial_{3} q^{\varepsilon, \pm}}{\varepsilon}|| Z_{3} U^{\varepsilon, \pm} \mid d x d \tau \\
& \leq C \int_{0}^{t}\left\|Z_{3} U^{\varepsilon, \pm}(\tau)\right\|_{L^{2}\left(\Omega^{ \pm}\right)}^{2} d \tau+C \int_{0}^{t}\left\|\frac{1}{\varepsilon} \nabla q^{\varepsilon, \pm}(\tau)\right\|_{L^{2}\left(\Omega^{ \pm}\right)} d \tau .
\end{aligned}
$$

From (46) and the fact that $\widehat{v}_{3}^{ \pm}=\widehat{B}_{3}^{ \pm}=0$, we have

$$
\frac{1}{\varepsilon} \nabla q^{\varepsilon, \pm}=-\partial_{t} u^{\varepsilon, \pm}-\left(\widehat{v}^{\prime \pm} \cdot \nabla^{\prime}\right) u^{\varepsilon, \pm}+\left(\widehat{B}^{\prime \pm} \cdot \nabla^{\prime}\right) h^{\varepsilon, \pm}+F_{1}^{ \pm} \text {. }
$$

Consequently, if we denote $Z_{0}=\partial_{t}, Z_{1}=\partial_{x_{1}}$, and $Z_{2}=\partial_{x_{2}}$, while $Z_{3}=\sigma \partial_{x_{3}}$ and use estimate (108), we obtain

$$
\begin{gathered}
\sum_{ \pm}\left\|\frac{1}{\varepsilon} \nabla q^{\varepsilon, \pm}(\tau)\right\|_{L^{2}\left(\Omega^{ \pm}\right)}^{2} \\
\leq C \sum_{ \pm}\left(\sum_{j=0}^{2}\left\|Z_{j} U^{\varepsilon, \pm}(\tau)\right\|_{L^{2}\left(\Omega^{ \pm}\right)}^{2}+\left\|F^{ \pm}(\tau)\right\|_{L^{2}\left(\Omega^{ \pm}\right)}^{2}\right) \\
\leq C \sum_{ \pm}\left(\left\|F^{ \pm}(\tau)\right\|_{L^{2}\left(\Omega^{ \pm}\right)}^{2}+\frac{e^{\tau / \alpha_{0}}}{\alpha_{0}} \sum_{j=0}^{2}\left\|Z_{j} U_{0}^{ \pm}\right\|_{L^{2}\left(\Omega^{ \pm}\right)}^{2}\right. \\
\left.+\frac{e^{\tau / \alpha_{0}}}{\alpha_{0}} \int_{0}^{\tau} \sum_{j=0}^{2}\left\|Z_{j} F^{ \pm}(s)\right\|_{L^{2}\left(\Omega^{ \pm}\right)}^{2} d s\right) .
\end{gathered}
$$

By combining such estimate with the previous ones for $I^{ \pm}$and $Z_{3} U^{\varepsilon, \pm}$, integrating by parts in order to simplify the double integral, and neglecting negative terms obtained in the righthand side, we deduce

$$
\begin{aligned}
\sum_{ \pm}\left\|Z_{3} U^{\varepsilon, \pm}(t)\right\|_{L^{2}\left(\Omega^{ \pm}\right)}^{2} \leq & \frac{C}{\alpha_{0}} \int_{0}^{t} \sum_{ \pm}\left\|Z_{3} U^{\varepsilon, \pm}(\tau)\right\|_{L^{2}\left(\Omega^{ \pm}\right)}^{2} d \tau \\
& +\frac{C}{\alpha_{0}} \sum_{ \pm} \beta^{ \pm}(t),
\end{aligned}
$$

where

$$
\begin{aligned}
\beta^{ \pm}(t)= & \left\|Z_{3} U_{0}^{ \pm}\right\|_{L^{2}\left(\Omega^{ \pm}\right)}^{2}+e^{t / \alpha_{0}} \sum_{j=0}^{2}\left\|Z_{j} U_{0}^{ \pm}\right\|_{L^{2}\left(\Omega^{ \pm}\right)}^{2} \\
& +\int_{0}^{t}\left(\left\|F^{ \pm}(\tau)\right\|_{L^{2}\left(\Omega^{ \pm}\right)}^{2}+\left\|Z_{3} F^{ \pm}(\tau)\right\|_{L^{2}\left(\Omega^{ \pm}\right)}^{2}\right) d \tau \\
& +e^{t / \alpha_{0}} \int_{0}^{t} \sum_{j=0}^{2}\left\|Z_{j} F^{ \pm}(\tau)\right\|_{L^{2}\left(\Omega^{ \pm}\right)}^{2} d \tau .
\end{aligned}
$$


By the Grönwall's lemma, we get

$$
\sum_{ \pm}\left\|Z_{3} U^{\varepsilon, \pm}(t)\right\|_{L^{2}\left(\Omega^{ \pm}\right)}^{2} \leq \frac{C e^{C t / \alpha_{0}}}{\alpha_{0}} \sum_{ \pm} \beta^{ \pm}(t) .
$$

Combining (107), (108), and (118), we obtain that

$$
\begin{aligned}
\sum_{ \pm}( & \left.\left\|U^{\varepsilon, \pm}(t)\right\|_{H_{*}^{1}\left(\Omega^{ \pm}\right)}^{2}+\left\|\partial_{t} U^{\varepsilon, \pm}(t)\right\|_{L^{2}\left(\Omega^{ \pm}\right)}^{2}\right) \\
\leq & \frac{C}{\alpha_{0}} e^{C t / \alpha_{0}} \sum_{ \pm}\left(\left\|U_{0}^{ \pm}\right\|_{H_{*}^{1}\left(\Omega^{ \pm}\right)}^{2}+\left\|\partial_{t} U_{0}^{ \pm}\right\|_{L^{2}\left(\Omega^{ \pm}\right)}^{2}\right) \\
& +\frac{C}{\alpha_{0}} e^{C t / \alpha_{0}} \sum_{ \pm} \int_{0}^{t}\left(\left\|F^{ \pm}(\tau)\right\|_{H_{*}^{1}\left(\Omega^{ \pm}\right)}^{2}+\left\|\partial_{t} F^{ \pm}(\tau)\right\|_{L^{2}\left(\Omega^{ \pm}\right)}^{2}\right) d \tau .
\end{aligned}
$$

To conclude, we need to estimate $f^{\varepsilon}$. From (87), proceeding as in the proof of Corollary 12 and using the previous estimate, we get

$$
\begin{aligned}
& \left\|u_{3}^{\varepsilon, \pm}(t)\right\|_{H^{1}\left(\Omega^{ \pm}\right)}^{2}+\left\|h_{3}^{\varepsilon, \pm}(t)\right\|_{H^{1}\left(\Omega^{ \pm}\right)}^{2} \leq C\left\|U^{\varepsilon, \pm}(t)\right\|_{1, * ; \Omega^{ \pm}}^{2}, \\
& \left\|f^{\varepsilon}(t)\right\|_{H^{1 / 2}(\Gamma)}^{2} \leq\left\|f_{0}\right\|_{H^{1 / 2}(\Gamma)}^{2} \\
& +\frac{C}{\alpha_{0}} e^{C t / \alpha_{0}} \sum_{ \pm}\left(\left\|U_{0}^{ \pm}\right\|_{1, * ; \Omega^{ \pm}}^{2}+\left[F^{ \pm}\right]_{1, *, t ; \Omega^{ \pm}}^{2}\right) .
\end{aligned}
$$

Adding up (119) and (121), we deduce the claim.

4.7. Conclusion. Let us denote by CP the compressible problem (49), (35), and (36) with $\left.q^{\varepsilon, \pm}\right|_{t=0} \equiv 0$ and by IP the incompressible problem (34), (35), and (36). Corollary 12 guarantees that, for $\varepsilon$ sufficiently small, there exists a unique solution

$$
\begin{aligned}
\left(U^{\varepsilon}, f^{\varepsilon}\right) \in & \mathscr{C}^{0}\left([0, T] ; H_{*}^{1}(\Omega)\right) \cap \mathscr{C}^{1}\left([0, T] ; L^{2}(\Omega)\right) \\
& \times \mathscr{C}^{0}\left([0, T] ; H^{1 / 2}(\Gamma)\right)
\end{aligned}
$$

to CP, and moreover, this solution satisfies (99), which implies, in particular, that $\left(u^{\varepsilon, \pm}, h^{\varepsilon, \pm}, f^{\varepsilon}\right)$ is bounded in $\left(H_{*}^{1}\left(\Omega^{ \pm}\right)\right)^{2} \times H^{1 / 2}(\Gamma)$, uniformly in $\varepsilon$ and $t \in[0, T]$. Thus, it is possible to extract a subsequence weakly convergent, as $\varepsilon \rightarrow$ 0 , to a certain $\left(u^{ \pm}, h^{ \pm}, f\right)$. Now, we will show that it is possible to find pressure terms $q^{ \pm}$(unique up to additive constants), so that $\left(q^{ \pm}, u^{ \pm}, h^{ \pm}, f\right)$ solves IP. Since we are considering linear systems, the weak limit can be performed easily, with the only exceptions of the singular term $(1 / \varepsilon) \nabla q^{\varepsilon, \pm}$ in (46) and the boundary condition $[q]=0$.

If we test (46) against a generic $\mathscr{C}^{\infty}$ divergence-free vector function $\varphi^{ \pm}$compactly supported in $Q_{T}^{ \pm}$, we observe that

$$
\frac{1}{\varepsilon} \int_{\Omega^{ \pm}} \nabla q^{\varepsilon, \pm} \cdot \varphi=-\frac{1}{\varepsilon} \int_{\Omega^{ \pm}} q^{\varepsilon, \pm} \operatorname{div} \varphi^{ \pm}=0
$$

and hence

$$
\partial_{t} u^{\varepsilon, \pm}+\left(\widehat{v}^{ \pm} \cdot \nabla\right) u^{\varepsilon, \pm}-\left(\widehat{B}^{ \pm} \cdot \nabla\right) h^{\varepsilon, \pm}-F_{1}^{ \pm}=0
$$

in the space of solenoidal distributions defined in $Q_{T}^{ \pm}$, that is to say, the left-hand side is a gradient $-\nabla Q^{\varepsilon, \pm}$ in the space of distributions. Taking the weak limit as $\varepsilon \rightarrow 0$, we obtain

$$
\partial_{t} u^{ \pm}+\left(\widehat{v}^{ \pm} \cdot \nabla\right) u^{ \pm}-\left(\widehat{B}^{ \pm} \cdot \nabla\right) h^{ \pm}-F_{1}^{ \pm}=-\nabla q^{ \pm}
$$

in a weak sense, where $q^{ \pm}(t) \in L_{\text {loc }}^{2}\left(\Omega^{ \pm}\right)$is unique up to additive constants, and $\nabla q^{ \pm}$is the weak limit of $\nabla Q^{\varepsilon, \pm}$. Note that $\nabla Q^{\varepsilon, \pm}$ converges since it is equal to a convergent quantity; moreover, its limit is still a gradient (by the fact that the subspace of gradients is closed). The weak formulation reads

$$
\begin{gathered}
\sum_{ \pm} \int_{0}^{T} \int_{\Omega^{ \pm}}\left(u^{ \pm} \cdot \partial_{t} \varphi+\left(\widehat{v}^{\prime \pm} \cdot \nabla^{\prime}\right) \varphi \cdot u^{ \pm}-\left(\widehat{B}^{\prime \pm} \cdot \nabla^{\prime}\right) \varphi \cdot h^{ \pm}\right. \\
\left.+F_{1}^{ \pm} \cdot \varphi+q^{ \pm} \operatorname{div} \varphi\right)=0
\end{gathered}
$$

for each $\varphi \in \mathscr{C}_{0}^{\infty}([0, T[\times \Omega)$ (space of compactly supported functions). Now, let us consider a generic $\varphi \in \mathscr{C}_{0}^{\infty}(] 0, T[\times \Omega)$; integrating by parts all terms but the ones with $F_{1}^{ \pm}$, we deduce that

$$
\begin{aligned}
& \sum_{ \pm} \int_{0}^{T} \int_{\Omega^{ \pm}}\left(\partial_{t} u^{ \pm}+\left(\widehat{v}^{\prime \pm} \cdot \nabla^{\prime}\right) u^{ \pm}-\left(\widehat{B}^{\prime \pm} \cdot \nabla^{\prime}\right) h^{ \pm}+\nabla q^{ \pm}-F_{1}^{ \pm}\right) \cdot \varphi \\
& \quad=-\int_{0}^{T} \int_{\Gamma}[q] \varphi_{3}
\end{aligned}
$$

(the other boundary terms are zero because of the support of $\varphi$ and the fact that the unit vectors normal to the front are constant and appear derived). Because of (125), the left-hand side is zero; this means that $[q]=0$ in $\omega_{T}$.

Passing to the limit $\varepsilon \rightarrow 0$ in (99), we get

$$
\begin{gathered}
\sum_{ \pm}\left(\left\|(u, h)^{ \pm}(t)\right\|_{1, * ; \Omega^{ \pm}}^{2}+\left\|\left(u_{3}, h_{3}\right)^{ \pm}(t)\right\|_{H^{1}\left(\Omega^{ \pm}\right)}^{2}\right)+\|f(t)\|_{H^{1 / 2}(\Gamma)}^{2} \\
\quad \leq C e^{C t} \sum_{ \pm}\left(\left\|\left(u_{0}, h_{0}\right)^{ \pm}\right\|_{1, * ; \Omega^{ \pm}}^{2}+\left[F^{ \pm}\right]_{1, *, t ; \Omega^{ \pm}}^{2}\right)+\left\|f_{0}\right\|_{H^{1 / 2}(\Gamma)}^{2} .
\end{gathered}
$$

Hence, $u, h \in L^{\infty}\left([0, T] ; H_{*}^{1}(\Omega)\right) \cap W^{1, \infty}\left([0, T] ; L^{2}(\Omega)\right), u_{3}$, $h_{3} \in L^{\infty}\left([0, T] ; H^{1}(\Omega)\right)$ and consequently $\left.\left(u_{3}, h_{3}\right)\right|_{\Gamma} \in L^{\infty}([0$, $T] ; H^{1 / 2}(\Gamma)$ ), while $f \in \mathscr{C}^{0}\left([0, T] ; H^{1 / 2}(\Gamma)\right)$ (continuity can be achieved from (72)).

The regularity of $\nabla q^{ \pm}$can be obtained from the first equation in (34):

$$
\begin{aligned}
\left\|\nabla q^{ \pm}(t)\right\|_{L^{2}\left(\Omega^{ \pm}\right)} \leq C( & \left\|u^{ \pm}(t)\right\|_{H_{*}^{1}\left(\Omega^{ \pm}\right)}+\left\|\partial_{t} u^{ \pm}(t)\right\|_{L^{2}\left(\Omega^{ \pm}\right)} \\
& \left.+\left\|h^{ \pm}(t)\right\|_{H_{*}^{1}\left(\Omega^{ \pm}\right)}+\left\|F_{1}^{ \pm}(t)\right\|_{L^{2}\left(\Omega^{ \pm}\right)}\right)
\end{aligned}
$$


(recall that $\widehat{v}_{3}^{ \pm}=\widehat{B}_{3}^{ \pm}=0$ ), where

$$
\begin{aligned}
\left\|F_{1}^{ \pm}(t)\right\|_{L^{2}\left(\Omega^{ \pm}\right)} & \leq\left\|F_{1}^{ \pm}(0)\right\|_{L^{2}\left(\Omega^{ \pm}\right)}+\int_{0}^{t}\left\|\partial_{t} F_{1}^{ \pm}(\tau)\right\|_{L^{2}\left(\Omega^{ \pm}\right)} d \tau \\
& \leq\left\|F_{1}^{ \pm}(0)\right\|_{L^{2}\left(\Omega^{ \pm}\right)}+\sqrt{t}\left[F_{1}^{ \pm}\right]_{1, *, t ; \Omega^{ \pm}}
\end{aligned}
$$

by the Cauchy-Schwarz inequality. Using (128), we finally conclude (39). This ends the proof of Theorem 2.

\section{References}

[1] L. D. Landau and E. M. Lifshitz, Course of Theoretical Physics. Vol. 8, Pergamon Press, Oxford, UK, 1984, Electrodynamics of continuous media, Translated from the second Russian edition by J. B. Sykes, J. S. Bell and M. J. Kearsley, Second Russian edition revised by Lifshits and L. P. Pitaevskiü.

[2] M. S. Ruderman and H. J. Fahr, "The effect of magnetic fields on the macroscopic instability of the heliopause. II. Inclusion of solar wind magnetic fields," Astronomy \& Astrophysics, vol. 299, pp. 258-266, 1995.

[3] D. Coutand and S. Shkoller, "Well-posedness of the freesurface incompressible Euler equations with or without surface tension," Journal of the American Mathematical Society, vol. 20, no. 3, pp. 829-930, 2007.

[4] D. Coutand and S. Shkoller, "Well-posedness in smooth function spaces for the moving-boundary three-dimensional compressible euler equations in physical vacuum," Archive for Rational Mechanics and Analysis, vol. 206, no. 2, pp. 515-616, 2012.

[5] A. Morando, Y. Trakhinin, and P. Trebeschi, "Stability of incompressible current-vortex sheets," Journal of Mathematical Analysis and Applications, vol. 347, no. 2, pp. 502-520, 2008.

[6] Y. Trakhinin, "On the existence of incompressible currentvortex sheets: study of a linearized free boundary value problem," Mathematical Methods in the Applied Sciences, vol. 28, no. 8, pp. 917-945, 2005.

[7] J.-F. Coulombel, A. Morando, P. Secchi, and P. Trebeschi, "A priori estimates for 3D incompressible current-vortex sheets," Communications in Mathematical Physics, vol. 311, no. 1, pp. 247275, 2012.

[8] P. Secchi and Y. Trakhinin, "Well-posedness of the linearized plasma-vacuum interface problem." Preprint.

[9] A. Morando, Y. Trakhinin, and P. Trebeschi, "Well-posedness of the linearized plasma-vacuum interface problem in ideal incompressible mhd," Quarterly of Applied Mathematics. In press.

[10] D. Lannes, "Well-posedness of the water-waves equations," Journal of the American Mathematical Society, vol. 18, no. 3, pp. 605-654, 2005.

[11] Y. Trakhinin, "Existence of compressible current-vortex sheets: variable coefficients linear analysis," Archive for Rational Mechanics and Analysis, vol. 177, no. 3, pp. 331-366, 2005.

[12] P. D. Lax and R. S. Phillips, "Local boundary conditions for dissipative symmetric linear differential operators," Communications on Pure and Applied Mathematics, vol. 13, pp. 427-455, 1960.

[13] P. Secchi, "Linear symmetric hyperbolic systems with characteristic boundary," Mathematical Methods in the Applied Sciences, vol. 18, no. 11, pp. 855-870, 1995.
[14] P. Secchi, "The initial-boundary value problem for linear symmetric hyperbolic systems with characteristic boundary of constant multiplicity," Differential and Integral Equations, vol. 9, no. 4, pp. 671-700, 1996.

[15] A. Morando and P. Secchi, "Regularity of weakly well posed hyperbolic mixed problems with characteristic boundary," Journal of Hyperbolic Differential Equations, vol. 8, no. 1, pp. 37-99, 2011.

[16] A. Morando, P. Secchi, and P. Trebeschi, "Regularity of solutions to characteristic initial-boundary value problems for symmetrizable systems," Journal of Hyperbolic Differential Equations, vol. 6, no. 4, pp. 753-808, 2009.

[17] P. Secchi, "Some properties of anisotropic Sobolev spaces," Archiv der Mathematik, vol. 75, no. 3, pp. 207-216, 2000.

[18] P. Secchi, "An initial boundary value problem in ideal magnetohydrodynamics," Nonlinear Differential Equations and Applications, vol. 9, no. 4, pp. 441-458, 2002.

[19] Y. Trakhinin, "The existence of current-vortex sheets in ideal compressible magnetohydrodynamics," Archive for Rational Mechanics and Analysis, vol. 191, no. 2, pp. 245-310, 2009.

[20] K. O. Friedrichs, "Symmetric positive linear differential equations," Communications on Pure and Applied Mathematics, vol. 11, pp. 333-418, 1958.

[21] Y. Shizuta, "The initial-boundary value problem for symmetric hyperbolic systems with characteristic boundary," in Qualitative aspects and applications of nonlinear evolution equations (Trieste, 1993), pp. 99-108, World Scientific Publishing, River Edge, NJ, USA, 1994. 


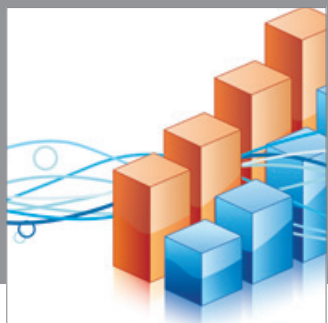

Advances in

Operations Research

mansans

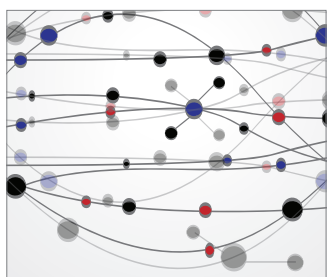

The Scientific World Journal
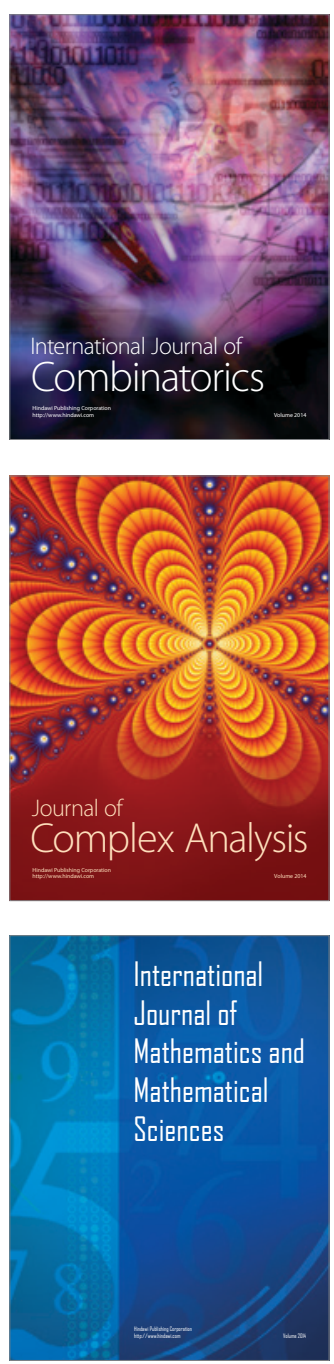
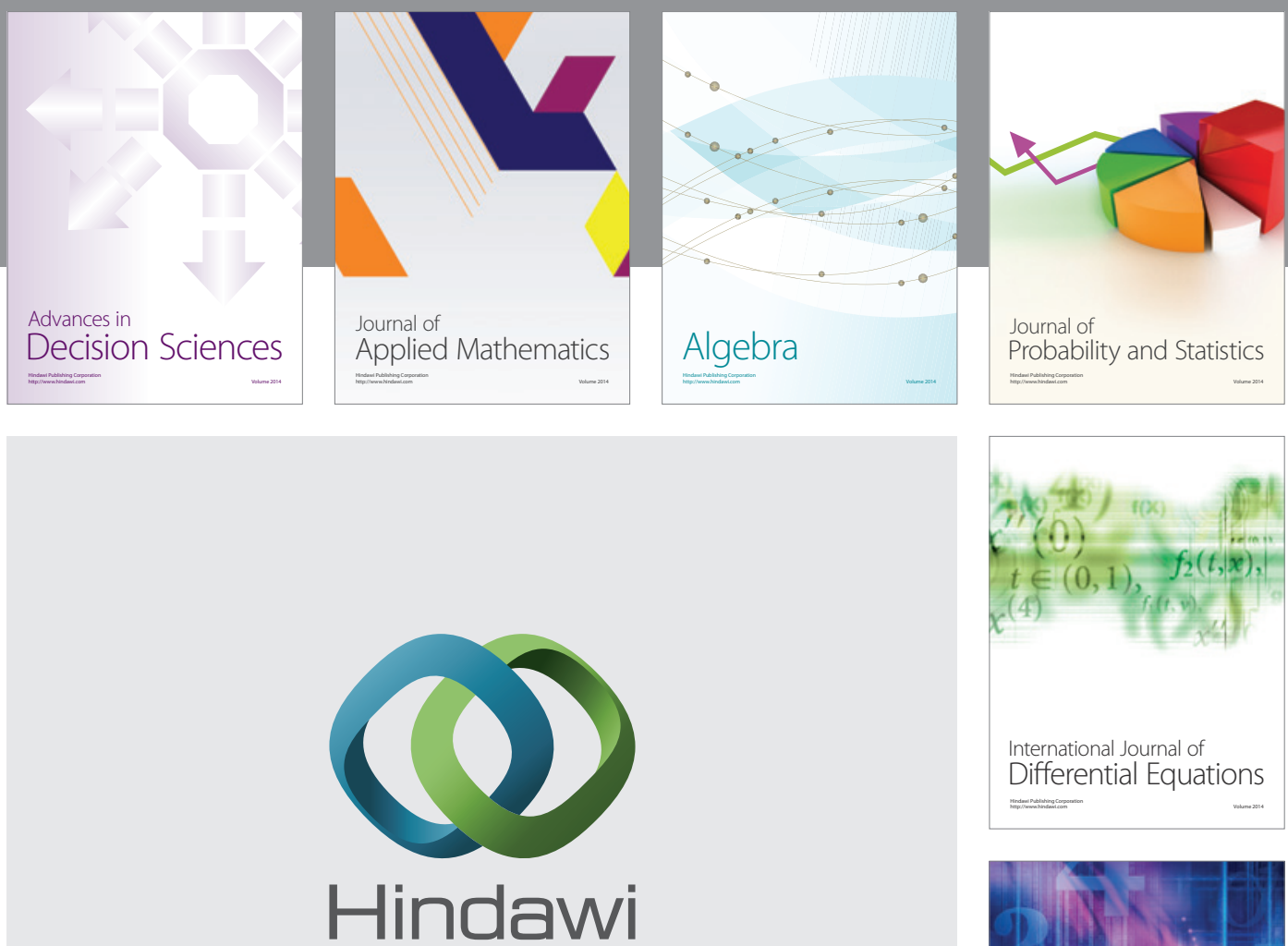

Submit your manuscripts at http://www.hindawi.com
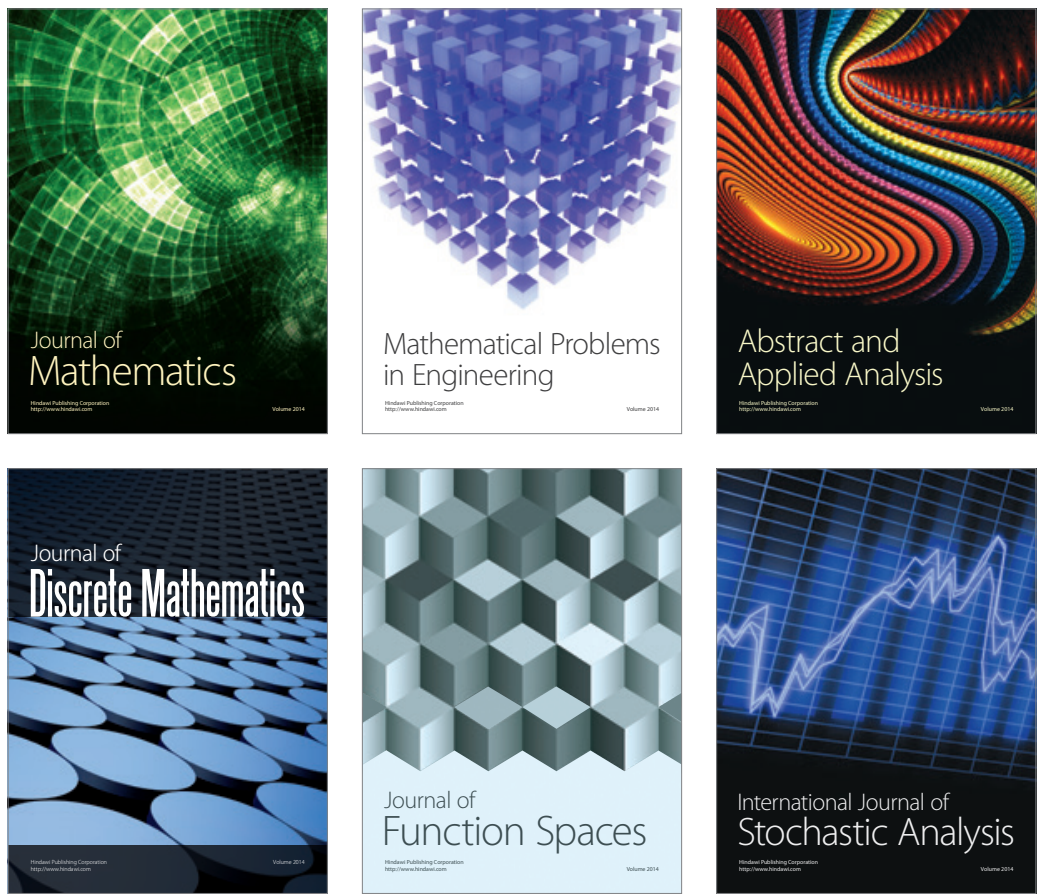

Journal of

Function Spaces

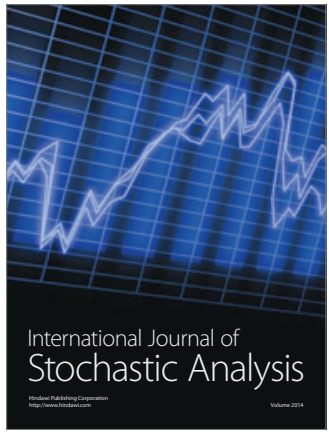

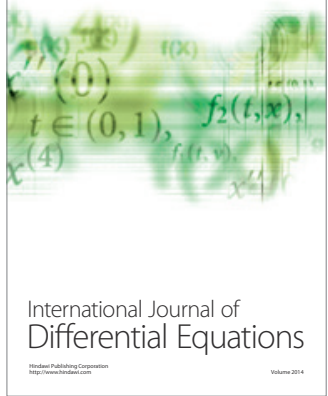
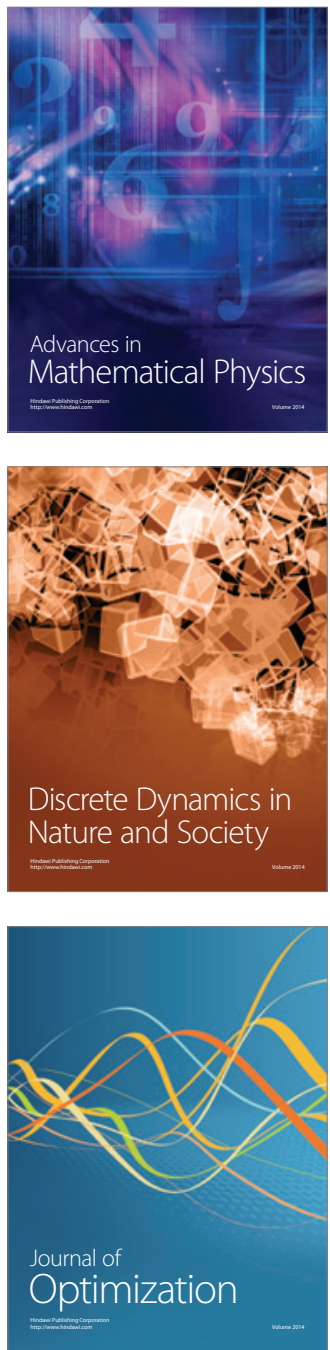